\title{
Stability of Perturbed Optimization Problems with Applications to Parameter Estimation
}

\author{
F. Coloniust \\ Institut für Dynamische Systeme \\ Universität Bremen \\ Bibliothekstrasse \\ D-2800 Bremen 33 \\ FRG
}

\author{
K. Kunisch* \\ Institut für Mathematik \\ Technische Universität Graz \\ Kopernikusgasse \\ A-8010 Graz \\ Austria
}

\section{Introduction}

The first objective of this paper is to study the stability of the solutions of the parameter dependent optimization problem

$(P)_{w} \min f(x, w)$ over $x \in Q_{a d} \subset Q$

with respect to changes in $w \in W$, where $W$ is a metric space. We consider the case where for $w \in W, f(\cdot, w)$ is defined on an open subset $D$ of the Banach space $Q$ with $D \supset Q_{a d}$, and stability is studied with respect to a (semi-)norm that is possibly different from the original norm on $\mathrm{Q}$. The necessity of considering two topologies arises because $\mathrm{x}$ may represent a parameter in a partial differential equation and hence it must enjoy sufficient regularity to allow for wellposedness of the partial differential equation. This determines the original topology on $\mathrm{Q}$. On the other hand, the nature of the optimization problem might not allow for stability of the solutions $x_{w}$ of $(P)_{W}$ with respect to this norm topology but rather only with respect to a weaker topology.

In section 2 we adapt techniques developed by W. Alt [Al] and $\mathrm{H}$. Maurer [M] to obtain stability results of the "two topology" optimization problem under a second order sufficient optimality condition. These results essentially assert that local solutions cannot vanish under perturbation and that they depend Hölder continuously on the perturbation parameter $w$ and secondly, that for every

* Supported in part by the Fonds zur Förderung der wissenschaftlichen Forschung, Österreich, S3206.

+ Supported by a Heisenberg Grant, Deutsche Forschungsgemeinschaft 
global solution of the perturbed problem $(\mathrm{P})_{\mathrm{W}}$ there must be a nearby global solution of the unperturbed problem $(\mathrm{P})_{\mathrm{w}^{0}}$ and that these global solutions also depend Hölder continuously on the parameter $w$.

In the subsequent sections these results are applied to parameter estimation problems associated with elliptic equations given by

$(P)_{z}$ minimize $|u(a)-z|_{Y}^{2}$ over $a \in Q_{a d}$

where $Y$ is an appropriately chosen Sobolov space, $z \in Y$, and $u=u(a)$ is the solution of

$$
\left\{\begin{array}{l}
-\operatorname{div}(a \operatorname{grad} u)+c u=f \text { on } \Omega \subset R^{\mathbf{n}} \\
u=0 \text { on } \partial \Omega
\end{array}\right.
$$

with $c, f \in L^{2}(\Omega)$ and $a \in Q_{a d}$, where

$$
Q_{a d}=\left\{a \in H^{2}(\Omega): a(x) \geq v(x)>0,|a|_{H^{2}} \leq \gamma\right\}, \text { with } v \in H^{2}(\Omega)
$$

For dimension $\mathrm{n}=1, \mathrm{H}^{2}(\Omega)$ is replaced by $\mathrm{H}^{1}(0,1)$. Concerning the stability of the solutions $\mathrm{a}_{\mathrm{z}}$ of $(P)_{z}$ with respect to changes in $z \in Y$, it is wellknown that $z \rightarrow z^{0}$ in $Y$ does not imply convergence of the solutions $a_{z}$ to $a_{z^{0}}$ in $H^{2}$ (resp. in $H^{1}$ if $n=1$ ), in general; see fur example

[Ru]. The question naturally arises whe :er it is possible to choose a weaker and problemdependent topology, for which $z \rightarrow z^{0}$ in $Y$ implies $a_{z} \rightarrow a_{z^{0}}$ in the weak topology.

In section 3 we take up this problem and suggest a solution for several parameter estimation problems associated with two point boundary value problems.

While the determination of this weak topology is of interest since it exhibits the inherent stability properties of an estimation problem associated with a partial differential equation, it might also indicate that for practical calculations the lack of stability with respect to a sufficiently fine topology for the parameter convergence cannot be ignored. One common remedy to this difficulty is the introduction of a regularization term. Thus we would replace $(\mathrm{P})_{z}$ by

$(P)_{z}^{\beta} \quad$ minimize $|u(a)-z|_{Y}^{2}+\beta\left(\sum_{i=1}^{n}\left|a_{x_{j}}\right|_{L^{2}}^{2}+\sum_{i, j=1}^{n}\left|a_{x_{i} x_{j}}\right|_{L^{2}}^{2}\right)$

where the regularization parameter $\beta$ is a small positive number. Observe that only a seminorm as opposed to the full $\mathrm{H}^{2}$-norm is used for regularization. This reflects our numerical experience with elliptic equations which shows that much better results are obtained with seminorms than with full norms. In section 4 we show that the solutions $\alpha_{z}^{\beta}$ of the regularized problems depend Hölder continuously (in $\mathrm{H}^{2}$-respectively $\mathrm{L}^{\infty}$ ) on $\mathrm{L}^{2}$-perturbations in the observation $\mathrm{z}$. We explicitly specify the Hölder constant in terms of $\beta, Q_{a d}$ and the distance of the unperturbed observation $z^{0}$ to the attainable set $V=\left\{u(a) ; a \in Q_{a d}\right\}$. As expected this constant diverges as $\beta \downarrow 0$. Explicit knowledge of the Hölder constant allows the study of the convergence of the solutions $a_{z}^{\beta}$ of the 
perturbed, regularized problems to the solutions $a_{z}^{0}$ of the unperturbed, unregularized problems.

For our analysis it is not necessary to require that the error free observation $z^{0}$ is contained in the attainable set $V$. We allow the possibility of modeling error, which is reflected in $\operatorname{dist}\left(z^{0}, V\right)>0$. However, if $\operatorname{dist}\left(\mathrm{z}^{0}, V\right)$ is too large then the range of admissible regularization parameters for which the regularized problems are stable, may shrink to zero. - In section 5 we address this problem and assert that if there is some model $S^{0}$ which has $z^{0}$ in its attainable set, then "nearby" models $S$ with modeling error still have good properties in the sense that there always exists a range of regularization parameter values for which the regularized problems for $S$ are stable and the solutions $\mathrm{a}_{\mathrm{z}}^{\beta}(S)$ of the regularized problems with observation error converge to solutions $\mathrm{a}_{\mathrm{z}}^{0} 0(S)$ of the observation error free unregularized problems for $S$.

Stability investigations which are comparable to those of the present paper were given in [C1,C2,C3, CK1,CK2, EKN,KS,N,Ri] for example. Chavent, [C1-C3] working in an abstract frame work studies the projection of $z^{0}$ onto the attainable set by geometric and Banach space analysis techniques. The results of Kravaris and Seinfeld [KS] are based on Tikhonov's lemma and Richter's approach in [Ri] builds upon the observation that when $u$ is replaced by $z^{0}$ equation (1.1) is a hyperbolic equation for a. In [EKN] and [N] various classical results from the wellknown theory of Tikhonov regularization for linear inverse problems are generalized to the nonlinear setting that is required for parameter estimation problems. These results are then applied to parameter estimation problems associated with two point boundary value problems and under appropriate assumptions on the a-priori knowledge of the solutions of $(P)_{z^{0}}^{0}$ rate of convergence for the solutions of the regularized problems to the solutions of the unregularized problem is proved.

The present research continues the investigations of [CK1,CK2]. It improves the earlier results by the fact that we can now work with two topologies, as explained above, and that we calculate explicitely the Hölder constant, whereas in [CK1,CK2] we only asserted the existence of such a constant. Now we can clarify the relation between the solutions of the regularized perturbed problems and the unregularized unperturbed problem (cp. Corollary 4.7, below). Moreover in this paper we consistently use only a seminorm for regularization as opposed to the full norm that was used in $[\mathrm{CK} 1, \mathrm{CK} 2]$.

Notation: By $H^{\mathrm{i}}(\mathrm{u})$ we denote the common Sobolev spaces over $\Omega$ with values in $\mathbf{R}$ as explained, for instance, in [Ad]. The space of essentially bounded functions endowed with the supremum norm is denoted by $L^{\infty}(\Omega)$. To specify the inner product and norm we generally use as an index the symbol of the Sobolev space, as for instance $|\varphi|_{H^{1}}$. However, we write $|\varphi|_{\infty}$ for $|\varphi|_{L^{\infty}}$ and with the $L^{2}(\Omega)=H^{0}(\Omega)$ inner product and norm we frequently drop the index.

In section 3 the function spaces are considered over bounded intervals $J \subset \mathbf{R}$. In this case $H^{i}(J)$, $i \in N$ is endowed with $\mid \varphi_{H^{i}(J)}=\left(\sum_{j=0}^{i}|\varphi(j)|_{L^{2}(J)}^{2}\right)^{1 / 2}$ as a norm. Moreover we put 
$\mathrm{H}_{0}^{1}(\mathrm{~J})=\left\{\varphi \in \mathrm{H}^{1}(\mathrm{~J}): \varphi(0)=\varphi(1)=0\right\}$ and use $|\varphi|_{\mathrm{H}_{0}^{1}(\mathrm{~J})}=\mid \varphi_{\mathrm{L}^{2}(\mathrm{~J})}$ as a norm for $\mathrm{H}_{0}^{1}(\mathrm{~J})$.

Similarly $\mathrm{H}_{0}^{2}(\mathrm{~J}):=\mathrm{H}_{0}^{1}(\mathrm{~J}) \cap \mathrm{H}^{2}(\mathrm{~J})$ is normed with $\left.\left|\varphi_{\mathrm{H}^{2}}{ }_{0}(\mathrm{~J})=\right| \varphi^{\prime \prime}\right|_{L^{2}(J)}$.

In section 3 - 5 free use of results from the theory of elliptic equations as explained in $[G, L U]$, for example, is made. Throughout differentiability is understood in the sense of Fréchetdifferentiability.

\section{Stability for Perturbed Optimization Problems}

In this section stability results for solutions of perturbed optimization problems are proved. The assumptions will allow applications to parameter estimation problems. We consider the family of problems

$(P)_{\mathrm{W}}$ minimize $f(x, w)$ such that $g(x) \in-K$,

where $f: D \times W \rightarrow R$ and $g: Q \rightarrow Y$, with $Q$ and $Y$ Banach spaces. Further $D$ is an open subset of $Q,(W, d)$ is a metric space and $K$ is a closed convex cone with vertex at the origin in $Y$. Keep $w^{0} \in W$ fixed. Then $w^{0}$ is used as the unperturbed reference value of the parameter $w$. The index $w$ will be dropped if no ambiguity can occur. We assume that

$$
Q_{\mathrm{ad}}=\{x \in Q: g(x) \in-K\}
$$

is a convex subset of $D$. A point $x^{0} \in Q_{a d}$ is called regular (with respect to the constraints) if

$$
0 \in \operatorname{int}\left\{g\left(x^{0}\right)+g_{x}\left(x^{0}\right) Q+K\right\}
$$

We assume throughout that $f$ and $g$ are twice continuously Fréchet differentiable with respect to $x$ and that $f$ is uniformly continuous with respect to $w$ at $w^{0}$; i.e. for all $\varepsilon>0$ there exists $\delta>$ 0 such that for all $x \in Q_{a d}$ and all $w \in W$

$$
d\left(w, w^{0}\right)<\delta \text { implies }\left|f(x, w)-f\left(x, w^{0}\right)\right|<\varepsilon
$$

We denote by $\varepsilon(\delta)$ the corresponding modulus of continuity. Thus for some $\delta_{0}>0$ and all $\delta \in\left(0, \delta_{0}\right)$

$$
\varepsilon(\delta):=\inf \left\{\varepsilon>0:(2.2) \text { holds for all } x \in Q_{a d}\right\}
$$

is welldefined and positive. Clearly $\varepsilon(\delta) \rightarrow 0^{+}$for $\delta \rightarrow 0^{+}$. Throughout we put $\mathrm{V}\left(\mathrm{w}^{0}\right)=\left\{w: \delta\left(w, w^{0}\right)<\delta_{0}\right\}$.

We recall the following first order necessary optimality condition (see e.g. [MZ; W, Theorem 5.3.2]). The dual cone $K^{*}$ of $K$ is given by $K=\left\{y^{*} \in Y^{*}:\left\langle y^{*}, y>\geq 0\right.\right.$ for all $\left.y \in K\right\}$. 
Proposition 2.1 Let $\mathrm{x}^{0}$ be a local solution of $(\mathrm{P}){ }_{\mathrm{w}^{0}}$ which is a regular point of $\mathrm{Q}_{\mathrm{ad}}$.

Then there exists a Lagrange multiplier $y^{*} \in Y^{*}$, i.e., $y^{*}$ satisfies

$$
f_{x}\left(x^{0}, w^{0}\right)+y^{*} g_{x}\left(x^{0}\right)=0, y^{*} \in K^{*}, y^{*} g\left(x^{0}\right)=0
$$

In order to prove a stability result for the solutions of $(P)_{W}$ the following second order sufficient optimality condition will be used. Recall that a seminorm $M$ on $Q$ is a mapping $M: Q \rightarrow R^{+}$ with $M(\beta x)=\beta M(x)$ for $\beta \in R^{+}$and $M(x+y) \leq M(x)+M(y)$.

(SSC) For a seminorm $M$ on $Q$ with $M(x) \leq m_{0}|x|$ for some $m_{0}$ independent of $x \in Q$, there exists $y^{*} \in Y^{*}$ satisfying (2.4) and for some $\alpha>0$ and $r>0$

$$
\left[f_{x x}\left(x, w^{0}\right)+y^{*} g_{x x}(x)\right]\left(v-x^{0}, v-x^{0}\right) \geq \alpha M\left(v-x_{0}\right)^{2}
$$

for all $v \in Q_{a d}$ and all $x \in Q_{a d}$ with $M\left(x-x^{0}\right) \leq r$.

Lemma 2.2 Condition (SSC) implies

$$
f\left(x, w^{0}\right) \geq f\left(x^{0}, w^{0}\right)+\alpha M\left(x-x^{0}\right)^{2}
$$

for all $x \in Q_{a d}$ with $M\left(x-x^{0}\right) \leq r$.

Proof: Let $x \in Q_{a d}$ with $M\left(x-x^{0}\right) \leq r$. Then $g(x) \in-K$ and hence $y^{*} g(x) \leq 0$. Thus

$$
f\left(x, w^{0}\right) \geq f\left(x, w^{0}\right)+y^{*} g(x),
$$

and, by the mean value theorem,

$$
\begin{aligned}
f\left(x, w^{0}\right) \geq f\left(x^{0}, w^{0}\right) & +y^{*} g\left(x^{0}\right) \\
& +\left[f_{x}\left(x^{0}, w^{0}\right)+y^{*} g_{x}\left(x^{0}\right)\right]\left(x-x^{0}\right) \\
& +\left[f_{x x}\left(\tilde{x}, w^{0}\right)+y^{*} g_{x x}(\tilde{x})\right]\left(x-x^{0}, x-x^{0}\right)
\end{aligned}
$$

with $\tilde{x}:=t x^{0}+(1-t) x, t \in[0,1]$. Now (SSC) is used to obtain

$$
\begin{aligned}
f\left(x, w^{0}\right) & \geq f\left(x^{0}, w^{0}\right)+\left[f\left(\tilde{x x}, w^{0}\right)+y^{*} g_{x x}\left(x^{0}\right)\right]\left(x-x^{0}, x-x^{0}\right) \\
& \geq f\left(x^{0}, w^{0}\right)+\alpha M\left(x-x^{0}\right)^{2},
\end{aligned}
$$

which is the desired estimate.

Lemma 2.3 Condition (SSC) implies

$$
f(x, w) \geq f\left(x^{0}, w^{0}\right)+\alpha M\left(x-x^{0}\right)^{2}-\varepsilon\left(d\left(w, w^{0}\right)\right)
$$

for all $x \in Q_{a d}$ with $M\left(x-x^{0}\right) \leq r$ and all $w \in V\left(w^{0}\right)$. 
Proof: Let $x \in Q_{a d}$ with $M\left(x \cdot x^{0}\right) \leq r$ and let $w \in V\left(w^{0}\right)$. Recalling (2.3) we have

$$
\left|f(x, w)-f\left(x, w^{0}\right)\right| \leq \varepsilon\left(d\left(w, w^{0}\right)\right) .
$$

From (SSC) and Lemma 2.2 one obtains

$$
f\left(x, w^{0}\right) \geq f\left(x^{0}, w^{0}\right)+\alpha M\left(x-x^{0}\right)^{2}
$$

and hence

$$
\begin{aligned}
f(x, w) & \geq f\left(x, w^{0}\right)-\left|f(x, w)-f\left(x, w^{0}\right)\right| \\
& \geq f\left(x^{0}, w^{0}\right)+\alpha M\left(x-x^{0}\right)^{2}-\varepsilon\left(d\left(w, w^{0}\right)\right) .
\end{aligned}
$$

The M-local extremal value function $\mu_{\mathrm{T}}(\cdot)$ is defined by

$$
\mu_{\mathrm{T}}(w):=\inf \left\{f(x, w): g(x) \in-K, M\left(x-x^{0}\right) \leq r\right\}
$$

Lemma 2.4 Let $x^{0}$ and $r>0$ be such that $f\left(x^{0}, w^{0}\right)=\mu_{r}\left(w^{0}\right)$. Then the M-local extremal value function $\mu_{\mathrm{r}}(w)$ is continuous at $w^{0}$ with the same modulus of continuity as $f$ with respect to w, i.e.

$$
\left|\mu_{T}(w)-\mu_{T}\left(w^{0}\right)\right| \leq \varepsilon\left(d\left(w, w^{0}\right)\right) \text { for } w \in V\left(w^{0}\right)
$$

with $\varepsilon(\cdot)$ given by $(2.3)$.

Proof: We first show that for all $w \in V\left(w^{0}\right)$

$$
\mu_{r}(w) \leq \mu_{r}\left(w^{0}\right)+\varepsilon\left(d\left(w, w^{0}\right)\right)
$$

By (2.3) we have for all $w \in V\left(w^{0}\right)$

$$
\begin{aligned}
\mu_{\mathrm{r}}(\mathrm{w}) & \leq \mathrm{f}\left(\mathrm{x}, \mathrm{w}^{0}\right) \\
& \leq f\left(\mathrm{x}^{0}, \mathrm{w}^{0}\right)+\left|\mathrm{f}\left(\mathrm{x}, \mathrm{w}^{0}\right)-\mathrm{f}\left(\mathrm{x}^{0}, \mathrm{w}^{0}\right)\right| \\
& \leq \mu_{\mathrm{r}}\left(\mathrm{w}^{0}\right)+\varepsilon\left(\mathrm{d}\left(\mathrm{w}, \mathrm{w}^{0}\right)\right) .
\end{aligned}
$$

On the other hand one obtains for all $x \in Q_{a d}$ with $M\left(x-x^{0}\right) \leq r$ and all $w \in V\left(w^{0}\right)$

$$
\begin{aligned}
\mu_{r}\left(w^{0}\right) & =f\left(x^{0}, w^{0}\right) \leq f\left(x, w^{0}\right) \\
& \leq f(x, w)+\left|f\left(x, w^{0}\right)-f(x, w)\right| \\
& \leq f(x, w)+\varepsilon\left(d\left(w, w^{0}\right)\right) .
\end{aligned}
$$

These two estimates imply the claim.

Theorem 2.5 Let $\mathrm{x}^{0} \in \mathrm{Q}_{\mathrm{ad}}$ satisfy (SSC). Suppose that (2.2) holds and that $\delta_{0}>0$ has been chosen small enough so that $E\left(\delta_{0}\right)<\alpha r^{2} / 2$. Suppose further that for all $w$ with $d\left(w, w^{0}\right) \leq \delta_{0}$ there is $x_{w} \in Q_{a d}$ with $M\left(x_{w}-x^{0}\right) \leq r$ and $\mu_{r}(w)=f\left(x_{w}, w\right)$.

Then $M\left(x_{w} \cdot x^{0}\right)<r$ for all $w$ satisfying $d\left(w, w^{0}\right) \leq \delta_{0}$, i.e. $x_{W}$ is a local minimum of $(P)_{w}$ (in the topology induced by the seminorm $M$ ) and 


$$
M\left(x_{w}-x^{0}\right) \leq\left(\frac{2}{\alpha}\right)^{1 / 2} \varepsilon\left(d\left(w, w^{0}\right)\right)^{1 / 2}
$$

Proof: By assumption $\mu_{\mathrm{r}}(w)=f\left(x_{w}, w\right)$ and from Lemma 2.2 we have $\mu_{r}\left(w^{0}\right)=f\left(x^{0}, w^{0}\right)$. Lemmas 2.3 and 2.4 imply

$$
\begin{aligned}
\alpha M\left(x_{w^{-}}-x^{0}\right)^{2} & \leq\left|f\left(x_{w}, w\right)-f\left(x^{0}, w^{0}\right)\right|+\varepsilon\left(d\left(w, w^{0}\right)\right) \\
& \leq\left|f\left(x_{w}, w\right)-\mu_{r}(w)\right|+\left|\mu_{r}(w)-\mu_{r}\left(w^{0}\right)\right|+\varepsilon\left(d\left(w, w^{0}\right)\right) \\
& \leq 2 \varepsilon\left(d\left(w, w^{0}\right)\right) \\
& \leq 2 \varepsilon\left(\delta_{0}\right) \\
& <\alpha r^{2} .
\end{aligned}
$$

Thus $\mathrm{M}\left(\mathrm{x}_{\mathrm{w}^{-}}-\mathrm{x}^{0}\right)<\mathrm{r}$ and also the asserted estimate (2.6) follows.

Remark 2.6 Suppose that $f$ is Lipschitz continuous w.r.t. $w$ at $w^{0}$ uniformly in $x \in Q_{a d}$, so that there exists $L>0$ such that

$$
\left|f(x, w)-f\left(x, w^{0}\right)\right| \leq L d\left(w, w^{0}\right)
$$

for all $x \in Q_{a d}$ and all $w$ in a neighborhood $V\left(w^{0}\right)$ of $w^{0}$. Then for $w \in V\left(w^{0}\right)$ estimate (2.6) has the form

$$
M\left(x_{w}-x^{0}\right) \leq\left(\frac{2 L}{\alpha}\right)^{1 / 2} d\left(w, w^{0}\right)^{1 / 2}
$$

since in this case $\varepsilon(\delta) \leq L \delta$. - An inspection of the proofs shows that it suffices that (2.7) holds uniformly w.r.t. only those $x \in Q_{a d}$ which satisfy $M\left(x^{0}-x\right) \leq r$ for $(2.6)^{\prime}$ to remain valid.

We proceed to discuss stability of globally optimal solutions.

Corollary 2.7 Let the following assumptions be satisfied:

(i) $\mathrm{Q}_{\mathrm{ad}}$ is weakly (sequentially) compact;

(ii) $Q$ has a compact embedding into a Banach space $X$ with norm $1 . \mathrm{I}_{X}$;

(iii) $\quad \mathrm{f}: \mathrm{D} \times \mathrm{W} \rightarrow \mathbf{R}$ is jointly continuous with respect to the $\mathrm{X}$-topology on $\mathrm{D}$ and the metric topology on $\mathrm{W}$;

(iv) There exist $\alpha>0$ and $r>0$ such that for every global solution $x^{0}$ of $(P)_{w^{0}}$ there exists $y^{*}=y^{*}\left(x^{0}\right) \in Y^{*}$ satisfying (2.4) and

$$
\left[f_{x x}\left(x, w^{0}\right)+y^{*} g_{x x}\left(x, w^{0}\right)\right](h, h) \geq \alpha|h|_{x}^{2}
$$


for all $h \in Q$ and all $x \in Q_{a d}$ with $\left|x \cdot x^{0}\right|_{X} \leq r$.

Then there exists $\delta_{1}>0$ such that for all $w \in W$ with $\mathrm{d}\left(\mathrm{w}, \mathrm{w}^{0}\right) \leq \delta_{1}$ and every global solution $x_{w}$ of $(P)_{w}$ there is a global solution $x^{0}$ of $(P)_{w^{0}}$ with

$$
\left|x_{w}-x^{0}\right|_{x} \leq\left(\frac{2}{\alpha}\right)^{1 / 2} \varepsilon\left(d\left(w, w^{0}\right)\right)^{1 / 2}
$$

Proof: First we show that there exists $\delta_{1}>0$ such that for all $w \in W$ with $d\left(w, w^{0}\right) \leq \delta_{1}$ and all global solutions $x_{w}$ of $(P)_{w}$ there is a global solution $x^{0}$ of $(P)_{w^{0}}$ with $\left|x_{w}-x^{0}\right|_{x}<r$. Suppose not. Then there exist a sequence $w^{n}$ converging to $w^{0}$ and global solutions $x_{n}$ of $(\mathrm{P})_{w^{\mathrm{n}}}$ such that

$$
\left|x_{n}-x^{0}\right|_{x} \geq I
$$

for all global solutions $x^{0}$ of $(P)_{w^{0}}$. By weak compactness of $Q_{a d}$ we may assume that $x_{n} \rightarrow x^{*}$ weakly in $Q$ and hence by (ii)

$$
\left|\mathrm{x}_{\mathrm{n}}-\mathrm{x}^{*}\right|_{\mathrm{X}} \rightarrow 0
$$

But this is impossible since it can be shown that $x^{*}$ is a global solution of $(P)_{w^{0}}$. In fact, by optimality of $x_{n}$ for $(P)_{w^{n}}$ one obtains

$$
f\left(x_{n}, w^{n}\right) \leq f\left(x, w^{n}\right) \text { for all } x \in Q_{a d}
$$

and with (iii)

$$
f\left(x^{*}, w^{0}\right) \leq f\left(x, w^{0}\right) \text { for all } x \in Q_{a d}
$$

Due to compacmess of $Q_{a d}$ in $X$ and as a consequence of (iii) the modulus of continuiy $\varepsilon(\delta)$ of $f$ w.r.t. $w$ uniformly in $x \in Q_{a d}$ is welldefined for $\delta$ sufficiently small. Decreasing $\delta_{1}$ if necessary, we can assume that $\varepsilon(\cdot)$ is defined on $\left[0, \delta_{1}\right]$. The proof can now be completed with an estimate analogous to that at the beginning of the proof of Theorem 2.5 .

Remark 2.8 If there exist $L>0$ and $\delta_{2}>0$ such that

$$
\left|f(x, w)-f\left(x, w^{0}\right)\right| \leq L d\left(w, w^{0}\right)
$$

for all $w \in W$ with $d\left(w, w^{0}\right)<\delta_{2}$ and all $x \in Q_{a d}$ with $\left|x-x^{0}\right|_{X} \leq r$, then (2.8) has the form

$$
\left|x_{w}-x^{0}\right|_{X} \leq\left(\frac{2 L}{\alpha}\right)^{1 / 2} d\left(w, w^{0}\right)^{1 / 2}
$$

for all $w \in W$ with $d\left(w, w^{0}\right) \leq \delta_{1}$ and $\delta_{1} \in\left(0, \delta_{2}\right)$.

Remark 2.9 The results and proofs of this section are similar to those in [AI]. The main difference between our treatment and that in [Al] lies in the fact that $Q$ is endowed with two different topologies here. The introduction of the second, weaker, topology realizes the fact that in certain classes of examples stability of the solutions does not hold with respect to the natural norm 
topology on $Q$ so that one is obliged to resort to some kind of weaker notion of stability. - The results in [Al] also admit perturbations of the admissible parameter set $\mathrm{Q}_{\mathrm{ad}}$.

This section is concluded with a technical lemma in which we give sufficient conditions on a point $x \in Q_{a d}$ to be regular in the sense of (2.1). It will be used frequently throughout the paper.

Let $L \subset Q$ be a closed convex cone with vertex at zero and let $q^{*}$ be a nontrivial bounded linear functional on $Q$ and denote with $\pi: Q \rightarrow \operatorname{ker} q^{*}$ the orthogonal projection from $Q$ onto the kernel of $q^{*}$. For $p \in Q, \mu \in \mathbf{R}$ and $\gamma \in \mathbf{R}^{+}$define

by

$$
\mathrm{g}: \mathrm{Q} \rightarrow \mathrm{Y}:=\mathrm{Q} \times \mathbf{R} \times \mathbf{R}
$$

and put $\mathrm{K}=\mathrm{L} \times \mathrm{R}^{+} \times\{0\} \subset \mathrm{Y}$

$$
g(x)=\left(p-x,|x|^{2}-\gamma^{2}, q^{*}(x)-\mu\right)
$$

Lemma 2.10 Let $\left[\text { ker } q^{*}\right]^{\perp} \cap L \neq\{0\}$ and assume that $q^{*}(L) \subset R^{+}, q^{*}(p)<\mu$ and $|\pi(p)|^{2}+\mu^{2}\left|h_{0}\right|^{2}<\gamma^{2}$ where $h_{0} \in\left(\text { ker } q^{*}\right)^{\perp} \cap L$ with $q^{*}\left(h_{0}\right)=1$. Then the set

$$
\mathrm{Q}_{\mathrm{ad}}:=\{x \in \mathrm{Q}: \mathrm{g}(\mathrm{x}) \in-\mathrm{K}\}
$$

is non-empty and every point $x$ of $Q_{a d}$ is regular i.e.,

$$
0 \in \operatorname{int}\left\{g(x)+g^{\prime}(x) Q+K\right\} \text { for all } x \in Q_{a d} .
$$

Proof: The existence of $h_{0}$ with the specified properties follows from [ker $\left.q^{*}\right]^{\perp} \cap L \neq\{0\}$ and $q^{*}(L) \subset R^{+}$. Moreover $Q$ can be expressed as $Q=\operatorname{ker} q^{*} \oplus \operatorname{span}\left\{h_{0}\right\}$. The orthogonal projection from $Q$ onto $\left[\operatorname{ker} q^{*}\right]^{\perp}$ which we denote by $\pi_{2}$ is given by

$$
\pi_{2}(x)=q^{*}(x) h_{0} \text { for } x \in Q \text {. }
$$

In order to show that $Q_{a d}$ is non-empty, we define

$$
\hat{\mathrm{x}}=\mathrm{p}+\left(\mu-\mathrm{q}^{*}(\mathrm{p})\right) \mathrm{h}_{0} \text {. }
$$

Observe that

$$
\begin{aligned}
& \mathrm{p}-\hat{\mathrm{x}}=\left(\mathrm{q}^{*}(\mathrm{p})-\mu\right) \mathrm{h}_{0} \in-\mathrm{L} \\
& |\hat{\mathrm{x}}|^{2}=|\pi(\mathrm{p})|^{2}+\mu^{2}\left|\mathrm{~h}_{0}\right|^{2}<\gamma^{2}
\end{aligned}
$$

and $\mathrm{q}^{*}(\hat{\mathrm{x}})=\mu$. Thus $\hat{\mathrm{x}} \in \mathrm{Q}_{\mathrm{ad}}$. Now let $\mathrm{x} \in \mathrm{Q}_{\mathrm{ad}}$ be chosen arbitrarily. We have to verify that

$$
0 \in \operatorname{int}\left\{\left(p-x-h+L,|x|^{2}-\gamma^{2}+2(x, h)+R^{+}, q^{*}(h)\right): h \in Q\right\} \subset Y
$$

Put

$$
\delta=\min \left(\frac{\gamma^{2}-|\pi(\mathrm{p})|^{2}-\mu^{2}\left|\mathrm{~h}_{0}\right|^{2}}{1+2 \gamma+2 \gamma\left|\mathrm{h}_{0}\right|}, \frac{\mu-\mathrm{q}^{*}(\mathrm{p})}{\| \mathrm{q}^{*} \mid+1}\right),
$$

where $\left\|q^{*}\right\|$ denotes the norm of $q^{*}$ and define $B=\left\{(\tilde{x}, \tilde{r}, \tilde{s}) \in Y:\|(\bar{x}, \tilde{r}, \tilde{s})\|_{Y} \leq \delta\right\}$. Without loss of generality we endow the product space with the supremum norm in this proof. We shall show that

(2.10) $B \subset\left\{\left(p-x-h_{1}-\sigma h_{0}+L,|x|^{2}-\gamma^{2}+2\left(x, h_{1}+\sigma h_{0}\right)+R^{+}, \sigma q^{*}\left(h_{0}\right)\right): h_{1} \in \operatorname{ker} q^{*}, \sigma \in \mathbf{R}\right\}$,

from which (2.9) follows. Let $(\tilde{\mathrm{x}}, \tilde{\mathrm{r}}, \tilde{\mathrm{s}}) \in \mathrm{B}$ be chosen arbitrarily. We put $\sigma=\tilde{\mathrm{s}}$ and verify that there exists $\left(h_{1}, l, r^{+}\right) \in$ ker $q^{*} \times L \times R^{+}$such that 


$$
\left(p-x-h_{1}-\tilde{s} h_{0}+1,|x|^{2}-\gamma^{2}+2\left(x, h_{1}+\tilde{s} h_{0}\right)+r^{+}\right)=(\tilde{x}, \tilde{r})
$$

This will imply the claim. Observe that by the choice of $\delta$ we find that $\mu-q^{*}(p)+q^{*}(\tilde{x})+\bar{s} \geq 0$. Hence 1 , defined by

$$
I=\left(\mu-q^{*}(p)+q^{*}(\tilde{x})+\tilde{s}\right) h_{0}
$$

is in $\mathrm{L}$. Choosing $h_{1}$ as

$$
\mathrm{h}_{1}=\pi(\mathrm{p}-\mathrm{x}-\tilde{\mathrm{x}})
$$

we obtain equality in the first coordinate of $(2.11)$. For the second coordinate in (2.11) w observe that

$$
\begin{aligned}
& |\mathrm{x}|^{2}-\gamma^{2}+2\left(\mathrm{x}, \mathrm{h}_{1}+\tilde{s} h_{0}\right)-\tilde{\mathrm{r}} \\
& =|\pi \mathrm{x}|^{2}+\mu^{2}\left|\mathrm{~h}_{0}\right|^{2}-\gamma^{2}+2\left(\mathrm{x}, \pi(\mathrm{p}-\mathrm{x}-\tilde{\mathrm{x}})+\tilde{s} h_{0}\right)-\tilde{\mathrm{r}} \\
& \left.\leq|\pi \mathrm{x}|^{2}+\mu^{2}\left|\mathrm{~h}_{0}\right|^{2}-\gamma^{2}+|\pi \mathrm{x}|^{2}+|\pi \mathrm{p}|^{2}-2|\pi \mathrm{x}|^{2}+2 \gamma|\tilde{x}|+|\tilde{s}|\left|\mathrm{h}_{0}\right|\right)-\tilde{\mathrm{r}} \\
& \leq \mu^{2}\left|\mathrm{~h}_{0}\right|^{2}-\gamma^{2}+|\pi \mathrm{p}|^{2}+2 \delta x\left(1+\left|\mathrm{h}_{0}\right|\right)+\delta .
\end{aligned}
$$

Hence there exists $r^{+} \in \mathbf{R}^{+}$such that equality holds in the second coordinate of (2.11). This ends the proof.

Remark 2.11 The conclusion of Lemma 2.10 remains valid if some of the constraints involved in defining $Q_{a d}$ are deleted. If the linear equality constraint is not present, then the assumptions $\mathrm{q}^{*}(\mathrm{p})<\mu$ and $|\pi \mathrm{p}|^{2}+\left|\mathrm{h}_{0}\right|^{2} \mu^{2}<\gamma^{2}$ are replaced by $|\mathrm{p}|<\gamma$.

\section{Stability for the Estimation Problem in a Natural Norm}

In this section we apply the results of section 2 to parameter estimation problems associated with elliptic boundary value problems. We consider

$$
-\left(\mathrm{au}_{\mathrm{x}}\right)_{\mathrm{x}}+\mathrm{cu}=\mathrm{f} \text { on }(0,1)
$$

for $f \in L^{2}$ together with appropriate boundary conditions. Unless otherwise specified, all function spaces are considered over the interval $(0,1)$ in this section. Let $q$ denote one of the coefficients a or $\mathrm{c}$ in (3.1) (the other one is assumed to be fixed). We assume that $\mathrm{q}$ is known outside of an interval $\mathrm{I}:=(\alpha, \beta) \subset[0,1]$; if $\alpha=0$ or $\beta=1$, these points are included in $\mathrm{I}$. Let $\mathrm{I}^{\mathrm{c}}$ denote $[0,1] \backslash \mathrm{I}$. The parameter estimation problem consists in determining the unknown coefficient $q$ over I from an observation $z$. This observation $z$ corresponds to the solution $u\left(q^{*}\right)$ of (3.1) evaluated at the "true", but unknown coefficients $q^{*}$. Due to modelling and observation error, $z$ may not coincide with $u\left(q^{*}\right)$. In this case $q$ is determined as the coefficient which gives the best fit of $u(q)$ to $z$. Thus we consider the least squares problem

$(\mathrm{P})_{z}$

$$
\min _{q \in Q_{a d}}|u(q)-z|_{z}^{2}
$$

where $\mathrm{Q}_{a d}=\mathrm{Q}_{\mathrm{ad}}(\mathrm{I})$ is the set of admissible parameters defined on I, with $\mathrm{Q}_{\mathrm{ad}} \subset \mathrm{Q}, \mathrm{Q}$ a Hilbert 
space, and $z$ is an element of the output space $Z$, which is also a Hilbert space. The space $Q$ is chosen such that its elements are sufficiently regular so that, together with lower pointwise bounds on $q$ guaranteeing ellipticity of the operator $A u=-\left(a u_{x}\right)_{x}+c u$, solutions $u(q)$ of (3.1) exist in $Z$. The observation $z$ is called attainable if $z$ is contained in the attainable set $V:=\left\{u(q): q \in Q_{a d}\right\}$. We shall not require attainability of $z$ in our analysis.

Henceforth we concentrate on the stability of the solutions of $(P)_{2}$ with respect to perturbations in the observation $z$. It is wellknown that $q \rightarrow u(q)$ from $Q_{a d} \subset Q$ to $Z$ is not continuously invertible (in a multivalued sense), in general [EKN,Ru]. This implies that the solutions $\mathrm{q}_{z}$ in $\mathrm{Q}_{\mathrm{ad}} \subset \mathrm{Q}$ of $(\mathrm{P})_{\mathrm{z}}$ do not depend continuously on $\mathrm{z}$, in general. In [CK1,CK2] we used Tikhonov regularization to define problems that are "near" to $(\mathrm{P})_{\mathrm{z}}$ and for which the solutions depend continuously on the observation $z$. Here we show that the problems $(P)_{z}$ themselves exhibit some weaker form of continuous dependence of the solutions $q_{z}$ of $(P)_{z}$ on the observations $z$ in $Z$. This will be accomplished by endowing $Q$ also with a coarser topology. Clearly, it is desirable for the gap between these two topologies to be small.

We denote by $z^{0} \in Z$ the unperturbed reference observation and suppose that

$$
Q \subset X, \quad X \text { a Banach space with norm }|\cdot|_{X} .
$$

Open neighborhoods of points $x$ in a Banach space are denoted by $V(x)$.

Definition 3.1 The parameter $q$ in $(P)_{z^{0}}$ is called output least squares stable (OLS-stable) with respect to $\left(Q_{a d}(I), X, Z\right)$ at the local solution $q_{z} 0$ of $(P)_{z} 0$ if there exist neighborhoods $V\left(z^{0}\right)$ in $Z$ and $V\left(q_{z}\right)$ in $X$ and a constant $k>0$ such that for all $z \in V\left(z^{0}\right)$ there exists a local solution $\mathrm{q}_{\mathrm{z}}$ of $(\mathrm{P})_{\mathrm{z}}$ in $\mathrm{V}\left(\mathrm{q}_{\mathrm{z}} 0\right)$ and for every such local solution $\mathrm{q}_{\mathrm{z}}$ of $(\mathrm{P})_{2}$ in $\mathrm{V}\left(\mathrm{q}_{\mathrm{z}}\right)$ we have

$$
\left|q_{z}-q_{z}\right|_{x} \leq k\left|z-z^{0}\right|_{z}^{1 / 2}
$$

We point out that - while we do not insist on uniqueness of the solutions of $(\mathrm{P})_{z} 0$ - the following local uniqueness property holds: If $\mathrm{q}_{\mathrm{z}}$ is a local solution of $(\mathrm{P})_{z^{0}}$ such that OLS-stability holds at $q_{z^{0}}$, then $q_{z^{0}}$ is locally (w.r.t. the X-topology) unique.

\section{(i) Estimation of the coefficient a}

We consider the estimation of the coefficient $a$ in

$$
\begin{aligned}
-\left(a u_{x}\right)_{x}+c u & =f \text { in }(0,1) \\
u(0)=u(1) & =0
\end{aligned}
$$

where $f \in L^{2}, f \neq 0$, and $c \in L^{2}, c \geq 0$ are fixed. 
Let $v \in H^{1}(0,1), \hat{a} \in H^{1}(0,1)$ and let $I=(\alpha, \beta) \subset[0,1]$ be as described above. Here $v$ will be used to give a lower bound on the set of admissible parameters and â plays the role of an a-priori guess for the true parameter. We consider only coefficients a which satisfy $a=v$ on $I^{c}$. Further it is assumed that

$$
\min v>0 \text { on }(0,1), \hat{a}=v \text { on } I^{c} \text { and } \int_{\alpha}^{\beta} v d x<m \text {, where } m=\int_{\alpha}^{\beta} \hat{a} d x \text {. }
$$

Two choices of admissible parameter sets will be considered:

$$
Q_{a d}^{2}=Q_{a d}^{2}(I)=\left\{a \in H^{1}(I): a \geq v \text { on } I,|a-\hat{a}|_{H^{1}(I)} \leq \gamma, a(\alpha)=v(\alpha), a(\beta)=v(\beta)\right\}
$$

and

$$
Q_{a d}^{1}=Q_{a d}^{1}(I)=\left\{a \in Q_{a d}^{2}: \int_{\alpha}^{\beta} a d x=m\right\}
$$

If $\alpha=0$ or $\beta=1$ the corresponding boundary condition in $Q_{a d}^{i}$ is omitted. Throughout it is assumed that

$$
|v-\hat{a}|_{H^{1}(1)}<\gamma
$$

Unless otherwise specified the elements a of $Q_{a d}^{i}, i=1,2$ are identified with their extensions to functions in $\mathrm{H}^{1}(0,1)$ obtained by $a(x):=v(x), x \in I^{c}$.

Due to the pointwise lower bound on the coefficient by the positive function $v$ there exists a unique solution $u(a) \in H_{0}^{1} \cap H^{2}$ of (3.2) for every $a \in Q_{a d}^{i}, i=1$ or 2 . One can show that $Q_{a d}^{i}$ is a closed and convex subset of $H^{1}(I)$ and hence it is weakly sequentially compact in $H^{1}(I)$. This implies that the problems

$$
\text { (P) } z^{0} \quad \min \left|u(a)-z^{0}\right|_{H_{0}^{i}} \text { over } Q_{a d}^{i}
$$

have a solution in $Q_{a d}^{i}$. The norm bound is essential for this argument. The remaining constraints that are involved in defining $Q_{a d}^{i}$ are required to guarantee OLS-stability of a local solution $a_{z} 0$ of the unperturbed problem $\left(\mathrm{P}_{\mathrm{z}^{0}}\right.$ and will be considered together with one of the following assumptions:

$$
\text { There exist constants } k_{1} \text { and } k_{2} \text { such that } 0<k_{1}<u_{x}\left(a_{z} 0\right)<k_{2}
$$$$
\text { on }[\alpha, \beta] \text { and } 0<\left(k_{2}-k_{1}\right)^{2}(\beta-\alpha)^{2}<k_{1}^{2} \text {. }
$$

There exist constants $k_{1}$ and $k_{2}$ such that $u_{x}\left(a_{z} 0\right)>k_{1}>0$

on $[\alpha, \beta],\left|u_{x x}\left(a_{z^{0}}\right)\right|_{L^{2}(\alpha, \beta)}<k_{2}$ and $2(\beta-\alpha) k_{2}^{2}<k_{1}^{2}$.

The problem $(P)_{z^{0}}$ over $Q_{a d}^{i}$ will be considered together with (Hi). Thus for the estimation of $\mathrm{a}$ in (3.2) from data $\mathrm{z}^{0}$ we fix the parameter $\mathrm{a}$ on $\mathrm{I}^{\mathrm{c}}$ as well as its mean on [0,1].

For $(\mathrm{H} 1)$ to hold, $u_{x}\left(a_{z^{0}}\right)$ should be large and flat over $[\alpha, \beta]$. 
Theorem 3.2 Assume that $a_{z^{0}}$ is a local solution of $(P)_{z} 0$, and that $z^{0} \in H_{0}^{1} \cap H^{2}$.

Then the following assertions hold.

(i) If (H1) is satisfied and if $\left|z_{x}^{0}-u_{x}\left(a_{z}\right)\right|_{\infty}$ is sufficiently small, then the coefficient a in $(P)_{z^{0}}$ is OLS-stable w.r.t. $\left(Q_{a d}^{1}(I), L^{2}(I), H_{0}^{1}\right)$ at $a_{z^{0}}$.

(ii) If (H2) is satisfied and $\left|z^{0}-u\left(a_{z^{0}}\right)\right|_{H_{0}^{2}}$ is sufficiently small, then the coefficient $a$ in $(P)_{z^{0}}$ is OLS-stable w.r.t. $\left(Q_{a d}^{2}(I), H^{1}(I), H_{0}^{2}\right)$ at $a_{z^{0}}$.

The proof of this and the following theorems is given at the end of this section.

We could not obtain an analogous result for $\mathrm{L}^{2}$ as an output topology; (technically, this appears to be due to the fact that the $\mathrm{H}^{-1}$ norm of the product of two functions cannot be bounded by the product of the corresponding $\mathrm{H}^{-1}$ norms).

While the $\mathrm{H}_{0}^{2}$ observation space might be of analytical value only, the $\mathrm{H}_{0}^{1}$ observation criterion proved to be numerically effective when $(P)_{z}$ was solved with an augmented Lagrangian method, even when only pointwise data were used and a discrete approximation to the least squares term $\left|u_{x}-z_{x}\right|_{L}^{2}$ was used [IK]. In the proof of Theorem 3.2, the condition on the distance of $u^{2}\left(a_{z}\right)$ to $z^{0}$ is given explicitly in terms of $k_{1}, k_{2}, f$ and $Q_{a d}^{i}$ (see (3.11), (3.17)).

\section{(ii) Estimation of the coefficient $c$}

We consider the estimation of the coefficient $c$ in (3.2) where $a \in H^{1}, a>0$ is fixed. Let $I$ be defined as above and let $v$ be a fixed element of $L^{2}$ satisfying $v \geq 0$ a.e. and $|v|_{L^{2}(I)}<\gamma$.

Define the set of admissible parameters by

$$
\mathrm{Q}_{\mathrm{ad}}:=\left\{c \in \mathrm{L}^{2}(\mathrm{I}): \mathrm{c} \geq v \text { on } \mathrm{I},|\mathrm{c}|_{\mathrm{L}^{2}(\mathrm{I})} \leq \gamma\right\}
$$

Unless otherwise specified the elements $c$ of $Q_{a d}$ are identified with their extensions to functions in $\mathrm{L}^{2}(0,1)$ obtained by

The problems

$$
c(x):=v(x), \quad x \in I^{c} .
$$

$$
(\mathrm{P})_{\mathrm{z}^{0}}^{\mathrm{i}} \min \left|\mathrm{u}(\mathrm{c})-\mathrm{z}^{0}\right|_{\mathrm{H}_{0}^{\mathrm{i}}} \text { over } \mathrm{Q}_{\mathrm{ad}}
$$

for $\mathrm{i}=1$ or $2, \mathrm{z}^{0} \in \mathrm{H}_{0}^{\mathrm{i}}$, have a solution $\mathrm{c}_{\mathrm{z}} \in \mathrm{Q}_{\mathrm{ad}}$.

The following assumption will be used:

$$
\text { There exists a constant } \left.k>0 \text { such that } u_{z^{0}}\right)>k \text { on }[\alpha, \beta] \text {. }
$$

Theorem 3.3 Assume that $\mathrm{c}_{z^{0}}$ is a local solution of $(\mathrm{P})_{z_{0}}^{\mathrm{i}}$ for $\mathrm{i}=1$ or 2 for which (H3) holds 
and that $\left|\mathrm{u}_{\mathrm{z}^{0}}\left(\mathrm{C}_{0}\right)-\mathrm{z}^{0}\right|_{\mathrm{H}_{0}^{\mathrm{i}}}$ is sufficiently small. Then the coefficient $\mathrm{c}$ in $(\mathrm{P})_{\mathrm{z}^{\mathrm{i}}}^{\mathrm{i}}$ is OLS-stable w.r.t. $\left(Q_{d d}(I), H^{i-2}(I), H_{D}^{i}\right)$ for $\mathrm{i}=1$ respectively 2 .

Proof of Theorem 3.2 The proof is given in several steps.

(i) Let us first summarize some facts that will be used in the sequel. There exist constants $\mathrm{K}_{1}$, $K_{2}$ and $K_{3}$ such that for $f \in L^{2}$ the following inequalities hold

$$
\frac{1}{\mathrm{~K}_{1}}|\mathrm{f}|_{\mathrm{L}^{2}} \leq|\mathrm{u}(\mathrm{a})|_{\mathrm{H}_{0}^{2}} \leq \mathrm{K}_{1}|\mathrm{f}|_{\mathrm{L}^{2}}
$$

$$
\frac{1}{\mathrm{~K}_{2}}|\mathrm{f}|_{\mathrm{H}^{-1}} \leq|\mathrm{u}(\mathrm{a})|_{\mathrm{H}_{0}^{1}} \leq \mathrm{K}_{2}|\mathrm{f}|_{\mathrm{H}^{-1}}
$$

and

$$
|u(a)|_{W^{1, \infty}} \leq K_{3} \sup _{x \in[0,1]}\left|\int_{0}^{x} f(s) d s\right|
$$

for all $a \in Q_{a d}^{i}, i=1$ or 2 . To verify (3.5) observe that by the mean value theorem for every $a \in Q_{a d}^{i}$ there exists $\xi \in[0,1]$ such that $u_{x}(\xi)=0$. Hence we obtain

$$
u_{x}=\frac{1}{a} \int_{\xi}^{x}(c u-f) d s
$$

and therefore by (3.4)

$$
\begin{aligned}
\sup _{x \in[0,1]}\left|u_{x}(x)\right| & \leq \frac{1}{\min v(x)}\left(|c|_{L^{2}}|u|_{L^{2}}+\sup _{x \in[0,1]}\left|\int_{\xi}^{x} f d s\right|\right) \\
& \leq \frac{1}{\min v(x)}\left(K_{1}|c|_{L^{2}}|f|_{H^{-1}}+2 \sup _{x \in[0,1]}\left|\int_{0}^{x} f d s\right|\right) .
\end{aligned}
$$

Since $|f|_{H^{-1}} \leq \sup _{x \in[0,1]}\left|\int_{0}^{x} f(s) d s\right|$, we easily obtain (3.5) from this estimate.

For $a \in H^{1}(0,1), a>0$, the mapping $a \rightarrow u(a)$ is twice continuously (Frechet-) differentiable from $H^{1}(0,1)$ to $H^{2}(0,1)$ and the first derivative $u_{a}(a)(h)$ in direction $h \in H^{1}(0,1)$, resp. the second derivative $u_{a a}(a)(h, h)$ in direction $(h, h) \in H^{1}(0,1) \times H^{1}(0,1)$ are characterized by

$$
A(a) \eta=\left\langle h u_{x}(a)\right)_{x} \text { and } A(a) \xi=2\left(h \eta_{x}\right)_{x}
$$

where $\eta=\eta(a ; h)=u_{a}(a)(h, h), \quad \xi=\xi(a ; h)=u_{a a}(a)(h, h)$ and $A(a): H_{0}^{1} \cap H^{2} \rightarrow L^{2}$ is given by $A(a) \varphi=-\left(a \varphi_{x}\right)_{x}+c \varphi$.

(ii) We show that the shifted set of admissible parameters

$$
\tilde{Q}_{a d}^{1}=\left\{a-\hat{a}: a \in Q_{a d}^{1}\right\}, i=1 \text { or } 2 \text {, }
$$


is non-empty and that every point in $\tilde{Q}_{a d}^{i}$ is regular. Let us first consider the case $i=1$ and $0<\alpha<\beta<1$. Then $\tilde{Q}_{a d}^{1} \subset \mathrm{Q}$ is given by

$$
\tilde{Q}_{a d}^{1}=\left\{a \in H^{1}(I): a \geq v-\hat{a},|a|_{H^{1}(I)} \leq \gamma, \int_{\alpha}^{\beta} a d x=0, a(\alpha)=a(\beta)=0\right\} .
$$

We apply Lemma 2.10 with

$\mathrm{Q}=\mathrm{H}_{0}^{1}(\mathrm{I})$ with $\left(|\mathrm{a}|_{L^{2}(\mathrm{I})}^{2}+|\dot{a}|_{L^{2}(\mathrm{I})}^{2}\right)^{1 / 2}$ as norm, $L=\{\mathrm{a} \in \mathrm{Q}: \mathrm{a} \geq 0\}, \mathrm{q}^{*}(\mathrm{a})=\int_{\alpha}^{\beta} \mathrm{a}(\mathrm{s}) \mathrm{ds}$, $\mathrm{p}=\nu-\hat{\mathrm{a}} \in \mathrm{Q}$ and $\mu=0$.

The mapping $\mathrm{g}: \mathrm{Q} \rightarrow \mathrm{Q} \times \mathbf{R} \times \mathbf{R}$ is given by $\mathrm{g}(\mathrm{a})=\left(\mathrm{p}-\mathrm{a},|\mathrm{a}|_{\mathrm{H}^{1}(\mathrm{I})}^{2}-\gamma^{2}, \mathrm{q}^{*}(\mathrm{a})\right)$.

Observe that $q^{*}(L) \subset \mathbf{R}^{+}, q^{*}(p)<m-\int_{\alpha}^{\beta} \hat{a} d x=0=\mu$ and

$|\pi \mathrm{p}|_{\mathrm{H}^{1}(\mathrm{I})}=|\pi(v-\hat{a})|_{H^{1}(\mathrm{I})} \leq|v-\hat{\mathrm{a}}|_{\mathrm{H}^{1}}<\gamma$. To apply Lemma 2.10 we need to argue

that $\left(\operatorname{ker} \mathrm{q}^{*}\right)^{\perp} \cap L \neq(0)$. Let $\psi$ be the unique solution of

$$
\left\{\begin{array}{c}
-\psi_{x x}+\psi=1 \text { on }(\alpha, \beta) \\
\psi(\alpha)=\psi(\beta)=0
\end{array}\right.
$$

By the maximum principle $\psi \in \mathrm{L}$. A short calculation shows that $\psi \in\left(\mathrm{ker}^{*}\right)^{\perp}$ and hence $h_{0}:=q^{*}(\psi)^{-1} \psi$ satisfies $h_{0} \in\left(\text { ker } q^{*}\right)^{\perp} \cap L$ and $q^{*}\left(h_{0}\right)=1$. Thus Lemma 2.10 implies the assertion in case $0<\alpha<\beta<1$ and $i=1$.

We now discuss the necessary changes when $0=\alpha<\beta<1$, and $i=1$. The case $0<\alpha<\beta=1$ is treated analogously and $0=\alpha<\beta=1$ cannot occur with (H1) holding. Thus $I=[0, \beta$ ) and

$$
\tilde{Q}_{a d}^{1}=\left\{a \in H^{1}(I): a \geq v-\hat{a},|a|_{H^{l}(I)} \leq \gamma, \int_{\alpha}^{\beta} a d x=0, a(\beta)=0\right\}
$$

We proceed as in the case $0<\alpha<\beta<1$ only with $Q$ chosen as $Q=\left\{a \in H^{1}(I): a(\beta)=0\right\}$ and $h_{0}=q^{*}(\psi)^{-1} \psi$, where $\psi$ is the unique solution of

$$
\left\{\begin{array}{c}
-\psi_{x x}+\psi=1 \text { on }(0, \beta) \\
\psi_{x}(0)=\psi(\beta)=0
\end{array}\right.
$$

In fact $\psi(t)=1-\left(e^{\beta}+e^{-\beta}\right)^{-1}\left(e^{t}+e^{-t}\right)$ and $\psi \in L \cap\left(\operatorname{ker} q^{*}\right)^{\perp} \cap Q$. The assertion again follows from Lemma 2.10.

Next consider the set $\tilde{\mathrm{Q}}_{\mathrm{ad}}^{2}=\left\{\mathrm{a}-\hat{\mathrm{a}}: \mathrm{a} \in \tilde{\mathrm{Q}}_{\mathrm{ad}}^{2}\right\}$. It is given by 


$$
\tilde{Q}_{a d}^{2}=\left\{a \in H^{1}(I): a \geq v-\hat{a},|a|_{H^{1}(I)} \leq \gamma, a(\alpha)=a(\beta)=0\right\}
$$

If $\alpha=0$ or $\beta=1$ the corresponding boundary condition in $\tilde{Q}_{\mathrm{ad}}^{2}$ is omitted. Since by assumption $|v-\hat{a}|_{H^{1}(I)}<\gamma$, it follows from Remark 2.11 that $\tilde{Q}_{a d}^{2}$ is non-empty and that each of its elements is a regular point.

(iii) We show that under the assumptions of Theorem 3.2(i) there exist constants $k>0$ and $r>0$ such that

$$
|\eta(a ; \stackrel{0}{h})|_{\mathrm{H}_{0}^{1}}^{2}+\left\langle\mathrm{u}(\mathrm{a})-\mathrm{z}^{0}, \xi(\mathrm{a} ; \stackrel{0}{\mathrm{~h}})\right\rangle_{\mathrm{H}_{0}^{1}} \geq \mathrm{k} \mid \stackrel{h}{h}_{L^{2}(\mathrm{l})}^{2},
$$

for all $a \in Q_{a d}^{1}$ with $\left|a-a_{z^{o}}\right|=\left|a-a_{z^{0}}\right|_{L^{2}(1)} \leq r$ and all $h \in H=\left\{h \in Q: \int_{\alpha}^{\beta} h d x=0\right\}$. Here $\stackrel{0}{h} \in \mathrm{H}^{1}$ is obtained as the extension of $h$ to $[0,1]$ by putting $\stackrel{0}{h}=0$ on $I^{c}$.

The two terms on the left hand side of (3.7) are estimated separately. We shall use the fact that the Laplacian $\Delta$ is an isometric isomorphism from $\mathrm{H}_{0}^{1}$ onto its dual $\mathrm{H}^{-1}$ and that $P=\mathrm{D} \Delta^{-1} \mathrm{D}$ can be extended to a bounded linear operator on $L^{2}$. Here $D$ stands for differentiation. Moreover $P$ is an orthogonal projection with $(\mathrm{I}-P) \varphi$ the constant function with value $\int_{0}^{1} \varphi \mathrm{ds}$ in $\mathrm{L}^{2}$, see e.g. [IK].

If $\left\{a_{n}\right\}$ is an arbitrary sequence in $Q_{a d}^{1}$ converging strongly in $L^{2}$ to an element $a^{*} \in Q_{a d}^{1}$, then $\left\{a_{n}\right\}$ converges weakly in $H^{1}$ to $a^{*}$, since $Q_{a d}^{1}$ is bounded in $H^{1}$. Consequently $u\left(a_{n}\right)$ converges weakly in $\mathrm{H}^{2}$ and hence strongly in $\mathrm{C}^{l}$ to $\mathrm{u}\left(\mathrm{a}^{*}\right)$. By $(\mathrm{HI})$ one can therefore choose $r_{1}$ such that $\left|a-a_{z^{0}}\right|_{L^{2}(I)} \leq r_{1}$ and $a \in Q_{a d}$ imply that $0<k_{1} \leq u_{x}(a) \leq k_{2}$ on $I$.

By (3.4) and (3.6) we find for $a \in Q_{a d}^{1}$ with $\left|a-a_{z^{0}}\right|_{L^{2}(I)} \leq r_{1}$ and $h \in H$

and therefore

$$
\begin{aligned}
|\eta(a ; \stackrel{0}{h})|_{H_{0}^{1}} & \left.=\mid A^{-1}(a) \stackrel{0}{h} u_{x}(a)\right)\left._{x}\right|_{H_{0}^{1}} \geq K_{2}^{-1}\left|\left(\stackrel{0}{h} u_{x}(a)\right)_{x}\right|_{H^{-1}} \\
& =K_{2}^{-1}\left|\Delta^{-1}\left(\stackrel{0}{h} u_{x}(a)\right)_{x}\right|_{H_{0}^{1}}=K_{2}^{-1}\left|P \stackrel{0}{h} u_{x}(a)\right|_{L^{2}}
\end{aligned}
$$

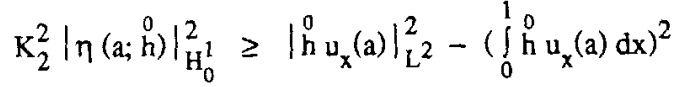

$$
\begin{aligned}
& =\left|h u_{x}(a)\right|_{L^{2}(I)}^{2}-\left(\int_{\alpha}^{\beta} h u_{x}(a) d x\right)^{2} .
\end{aligned}
$$

To estimate the last term we put

Then we obtain

$$
\Omega^{+}=\{x \in(\alpha, \beta): h(x) \geq 0\} \text { and } \Omega^{-}=\{x \in(\alpha, \beta): h(x)<0\}
$$

$$
\int_{\alpha}^{\beta} h u_{x}(a) d x=\int_{\Omega^{+}} h u_{x}(a) d x+\int_{\Omega^{-}} h u_{x}(a) d x \leq k_{2} \int_{\Omega^{+}} h d x+k_{1} \int_{\Omega^{-}} h d x
$$




$$
=k_{2} \int_{\alpha}^{\beta} h d x+\left(k_{1}-k_{2}\right) \int_{\Omega^{-}} h d x=\left(k_{1}-k_{2}\right) \int_{\Omega^{-}} h d x \leq\left(k_{2}-k_{1}\right) \int_{\alpha}^{\beta}|h| d x .
$$

This estimate is used in (3.8)

$$
\left.\mathrm{K}_{2}^{2} \ln (\mathrm{a} ; \mathrm{h})\right|_{\mathrm{H}_{0}^{1}} ^{2} \geq \mathrm{k}_{1}^{2}|\mathrm{~h}|_{\mathrm{L}^{2}(\mathrm{I})}^{2}-\left.\left(\mathrm{k}_{1}-\mathrm{k}_{2}\right)^{2} \ln \right|_{\mathrm{L}^{2}(\mathrm{D})}(\beta-\alpha)^{2}
$$

and thus we find that

$$
|\eta(a ; \stackrel{0}{h})|_{H_{0}^{1}}^{2} \geq K_{2}^{-2}\left[k_{1}^{2}-\left(k_{1}-k_{2}\right)^{2}(\beta-\alpha)^{2}\right]|h|_{L^{2}(1)}^{2}
$$

for all $a \in Q_{a d}$ with $\left|a-a_{z} 0\right|_{L^{2}(I)}^{2} \leq r_{1}$ and all $h \in H$.

We turn to the second term on the left hand side of (3.7).

For $\mathrm{a} \in \mathrm{Q}_{\mathrm{ad}}^{1}$ and $\mathrm{h} \in H$ we obtain by (3.3)-(3.6)

$$
\begin{aligned}
& \mid\left\langle u(a)-z^{0}, \xi(a ; \stackrel{0}{h}){ }_{H_{0}^{1}}|=2|\left\langle\left(A^{-1}(a) \Delta\left(u(a)-z^{0}\right)\right)_{x}, h^{0} \eta_{x}(a ; \stackrel{0}{h})\right\rangle\right| \\
& \leq 2\left|\left(\mathrm{~A}^{-1}(\mathrm{a}) \Delta\left(\mathrm{u}(\mathrm{a})-\mathrm{z}^{0}\right)\right)_{\mathrm{x}}\right|_{\infty}\left|\stackrel{\mathrm{h}}{\mathrm{h}}^{0}\right|\left|\eta_{\mathrm{x}}(\mathrm{a} ; \mathrm{h})\right| \\
& \leq 2 \mathrm{~K}_{3} \sup _{\mathrm{x} \in(0,1)}\left|\int_{0}^{\mathrm{x}}\left[\Delta\left(\mathrm{u}(\mathrm{a})-\mathrm{z}^{0}\right)\right](\mathrm{s}) \mathrm{ds}\right|\left|\mathrm{h}_{\mathrm{h}}^{0}\right|\left|\eta_{\mathrm{x}}(\mathrm{a} ; \mathrm{h})\right| \quad \text { (by (3.5)) } \\
& \leq\left. 4 \mathrm{~K}_{2} \mathrm{~K}_{3}\left|\mathrm{u}_{\mathrm{x}}(\mathrm{a})-\mathrm{z}_{\mathrm{x}}^{0}\right|_{\infty}|\stackrel{0}{\mathrm{~h}}|\right|_{\mathrm{h}} ^{0} \mathrm{u}_{\mathrm{x}}(\mathrm{a}) \mid \\
& \leq 4 \mathrm{~K}_{1} \mathrm{~K}_{2} \mathrm{~K}_{3}|\mathrm{f}| \mathrm{u}_{\mathrm{x}}(\mathrm{a})-\left.\mathrm{z}_{\mathrm{x}}^{0}\right|_{\infty}|\mathrm{h}|_{\mathrm{L}^{2}(\mathrm{I})}^{2}
\end{aligned}
$$

Let us assume that

$$
\left|u_{x}\left(a_{z}{ }^{\alpha}\right)-z_{x}^{0}\right|_{\infty}<\frac{K_{2}^{-2}\left[k_{1}^{2}-\left(k_{1}-k_{2}\right)^{2}(\beta-\alpha)^{2}\right]}{4 K_{1} K_{2} K_{3}|f|} .
$$

Since $a \rightarrow u_{x}(a)$ from $Q_{a d}^{1}$, endowed with the $L^{2}$-topology to $L^{\infty}$ is continuous, there exists $r \in\left(0, r_{1}\right)$ such that (3.11) with $a_{z^{0}}$ replaced by a holds uniformily for all $a \in Q_{a d}$ with $\left|a-a_{z^{0}}\right| \leq r$. From (3.9) and (3.10) we deduce the existence of a constant $k>0$ such that

$$
\left.\ln (\mathrm{a} ; \mathrm{h})\right|_{\mathrm{H}_{0}^{1}} ^{2}+\left\langle\mathrm{u}(\mathrm{a})-\mathrm{z}^{0}, \xi(\mathrm{a} ; \stackrel{0}{\mathrm{~h}})\right\rangle_{\mathrm{H}_{0}^{1}} \geq \mathrm{k}|\mathrm{h}|_{\mathrm{L}^{2}(\mathrm{I})}^{2}
$$

for all $\mathrm{a} \in \mathrm{Q}_{\mathrm{ad}}^{1}$ with $\left|\mathrm{a}_{\mathrm{z}^{0}}-\mathrm{a}\right|_{\mathrm{L}^{2}(\mathrm{l})} \leq \mathrm{r}$ and all $\mathrm{h} \in H$, provided that (3.11) holds.

(iv) We verify Theorem 3.2(i). It can be seen that (2.2) is satisfied. Let $E: \tilde{Q}_{a d}^{1} \rightarrow Q_{a d}^{1}$ be given by $\mathrm{E}(\tilde{\mathrm{a}})=\overrightarrow{\mathrm{a}}+\hat{\mathrm{a}}$. Clearly $\mathrm{E}$ is a homomorphism when $\tilde{\mathrm{Q}}_{\mathrm{ad}}^{1}$ and $\mathrm{Q}_{\mathrm{ad}}^{1}$ are endowed with the $\mathrm{H}^{1}$-topology. The problem

$(\mathrm{P})_{\mathrm{z}} \quad \min |\mathrm{u}(\mathrm{a})-\mathrm{z}|_{\mathrm{H}_{0}^{1}}^{2}$ over $\mathrm{Q}_{\mathrm{ad}}^{1}$ 
is equivalent to

$(\tilde{\mathrm{P}})_{\mathrm{z}} \quad \min |\mathrm{u}(\mathrm{E}(\tilde{\mathrm{a}}))-\mathrm{z}|_{\mathrm{H}_{0}^{1}}^{2}$ over $\tilde{\mathrm{Q}}_{\mathrm{ad}}^{1}$

in the sense that $\tilde{a}_{z}$ is a local solution of $(\tilde{\mathrm{P}})_{z}$ if and only if $E\left(\tilde{a}_{z}\right)$ is a local solution of $(P)_{z}$.

(Both, in $(P)_{z}$ and $(\tilde{P})_{z}$ it is understood that a resp. $E(\tilde{a})$ are extended to $(0,1)$ by setting the coefficient equal to $v$ there). Let $\tilde{a}_{z^{0}}=a_{z^{0}}-\hat{a}$ with $a_{z^{0}}$ as in the statement of Theorem $3.2(i)$.

We show that for $\mathrm{f}: \overline{\mathrm{Q}}_{\mathrm{ad}}^{1} \rightarrow \mathrm{H}^{1}$ given by

$$
f(\tilde{a})=\left|u(E(\tilde{a}))-z^{0}\right|_{H_{0}^{1}}^{2}
$$

condition (SSC) is satisfied so that Theorem 2.5 is applicable. This will imply Theorem 3.2 (i).

By (ii) and Proposition 2.1 there exists a Lagrange multiplier $y^{*}=\left(y_{1}^{*}, y_{2}^{*}, y_{3}^{*}\right) \in Q \times \mathbf{R} \times \mathbf{R}$ satisfying (2.4). Hence it suffices to argue uniform positivity of the second Fréchet derivative of $f+y^{*} g$. Observe that for every $\tilde{a} \in Q_{a d}^{1}$ and $h \in Q$

$$
\left.<y^{*}, g_{a a}(\tilde{a})(h, h)\right\rangle_{H^{1}(I) \times R}=y_{2}^{*} \mid h_{H^{1}(I)}^{2} \geq 0,
$$

since $\mathrm{y}_{2}^{*} \geq 0$. For all $\tilde{\mathrm{a}} \in \mathrm{Q}_{\mathrm{ad}}^{1}$ and $\mathrm{h} \in \mathrm{Q}$ we find

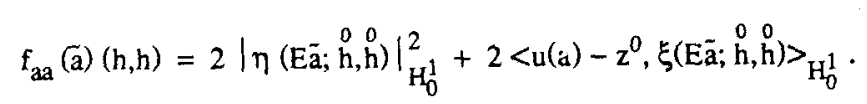

From (3.7) we conclude that

$$
f_{\mathrm{aa}}(\mathrm{a})(h, h) \geq \frac{k}{2}|h|_{L^{2}(1)}^{2}
$$

for all $\tilde{a} \in \tilde{Q}_{a d}^{1}$ with $\left|E \tilde{a}-a_{z^{0}}\right|_{L^{2}(\mathrm{I})}=\left|\vec{a}-\bar{a}_{z^{0}}\right|_{L^{2}(I)} \leq \mathrm{r}$ and all $\mathrm{h} \in H$.

Therefore we obtain

$f_{a a}(\tilde{a})(h, h)+y^{*} g_{a a}(\tilde{a})(h, h) \geq \frac{k}{2}|h|_{L^{2}(I)}^{2}$ for all $h \in H$ and $\tilde{a} \in \tilde{Q}_{a d}^{1}$ with $\left|\tilde{a}-\bar{a}_{z^{0}}\right|_{L^{2}(I)} \leq r$, and Theorem 2.5 implies Theorem 3.2(i).

(v) We now prove part (ii). First we ascertain the existence of constants $k>0$ and $r>0$ such that

$$
\begin{aligned}
& \left.\left.\ln (a ; \stackrel{0}{h})\right|_{H_{0}^{2}} ^{2}+<u(a)-z^{0} ; \xi(a ; h)\right\rangle_{H_{0}^{2}} \geq k\left|h_{H^{1}(I)}^{0}\right|^{2} \\
& \text { for all } a \in Q_{a d}^{2} \text { with }\left|a-a_{z^{0}}\right|_{H^{l}(I)} \leq I \text { and all } h \in Q \text {. }
\end{aligned}
$$

By $\left(H_{2}\right)$ one can choose $r_{1}>0$ such that $\left|a-a_{z}\right|_{H^{1}} \leq r_{1}$ and $a \in Q_{a d}^{2}$ imply that

$$
u_{x}(a) \geq k_{1} \text { on I and }\left|u_{x x}(a)\right|_{L^{2}(I)} \leq k_{2} \text {. }
$$

Using (3.3) and (3.6) we obtain for $a \in Q_{a d}^{2}$ with $\left|a-a_{z^{0}}\right|_{H^{1}} \leq r_{1}$ and $h \in Q$ 


$$
\begin{aligned}
& \left.|\eta(a ; \stackrel{0}{h})|_{H_{0}^{2}}^{2}=\mid A^{-1}(a) \stackrel{0}{h} u_{x}(a)\right)\left._{x}\right|_{H_{0}^{2}} ^{2} \geq K_{1}^{-2}\left|\left(h u_{x}(a)\right)_{x}\right|_{L^{2}(I)}^{2} \\
& =K_{1}^{-2}\left|h u_{x x}(a)+h_{x} u_{x}(a)\right|_{L^{2}(I)}^{2} \geq K_{1}^{-2} \int_{I}\left(\frac{1}{2}\left|h_{x} u_{x}\right|^{2}-\left|h u_{x x}\right|^{2}\right) d x \\
& =\frac{K_{1}^{-2} k_{1}^{2}}{2} \int_{I}\left|h_{x}\right|^{2} d x-K_{1}^{-2}|h|_{\infty}^{2}\left|u_{x x}\right|_{L^{2}(I)}^{2} \geq K_{1}^{-2}\left(\frac{k_{1}^{2}}{2}-(\beta-\alpha) k_{2}^{2}\right)\left|h_{x}\right|_{L^{2}(I)}^{2}
\end{aligned}
$$

We turn to the second term on the left hand side of (3.14). For $a \in Q_{z d}^{2}$ and $h \in Q$ we obtain by (3.3) and (3.6)

$$
\begin{aligned}
& \left|<\mathrm{u}(\mathrm{a})-\mathrm{z}^{0}, \xi(\mathrm{a} ; \stackrel{0}{\mathrm{~h}})\right\rangle_{\mathrm{H}_{0}^{2}}|\leq 2| \mathrm{u}(\mathrm{a})-\left.\mathrm{z}^{0}\right|_{\mathrm{H}_{0}^{2}}\left|\mathrm{~A}^{-1}(\mathrm{a})\left(\stackrel{0}{\mathrm{~h}} \eta_{\mathrm{x}}(\mathrm{a} ; \stackrel{0}{\mathrm{~h}})\right)_{\mathrm{x}}\right|_{\mathrm{H}_{0}^{2}} \\
& \leq 2 \mathrm{~K}_{1}\left|\mathrm{u}(\mathrm{a})-\mathrm{z}^{0}\right|_{\mathrm{H}_{0}^{2}}\left(\left|\stackrel{0}{h} \eta_{\mathrm{xx}}\left(\mathrm{a} ; \mathrm{h}^{\mathrm{h}}\right)\right|_{\mathrm{L}^{2}}+\left.||_{\mathrm{h}}^{0} \eta_{\mathrm{x}}\right|_{\mathrm{L}^{2}}\right) \\
& \leq 2 K_{1}\left|\eta_{1}(a)-z^{0}\right|_{H_{0}^{2}}\left(\left|h_{h}^{0}\right|_{\infty}\left|\eta_{x x}\right|_{L^{2}}+\left|h_{x}^{0}\right|_{L^{2}} 2\left|\eta_{x}\right|_{\infty}\right) \\
& \left.\leq 4 \mathrm{~K}_{1}\left|\mathrm{u}(\mathrm{a})-\mathrm{z}^{0}\right|_{\mathrm{H}_{0}^{2}}\left|\mathrm{~h}_{\mathrm{x}}\right|_{\mathrm{L}^{2}(\mathrm{I})}|\eta|_{\mathrm{H}_{0}^{2}}\right) \\
& \leq 4 \mathrm{~K}_{1}^{2}\left|\mathrm{u}(\mathrm{a})-\mathrm{z}^{0}\right|_{\mathrm{H}^{2}}\left|\mathrm{~h}_{\mathrm{x}}\right|_{\mathrm{L}^{2}(\mathrm{I})}\left|\left(\mathrm{h} \mathrm{u}_{\mathrm{x}}(\mathrm{a})\right)_{\mathrm{x}}\right|_{\mathrm{L}^{2}(\mathrm{I})} \\
& \leq 8 \mathrm{~K}_{1}^{2}\left|\mathrm{u}(\mathrm{a})-\mathrm{z}^{0}\right|_{\mathrm{H}_{0}^{2}}\left|\mathrm{~h}_{\mathrm{x}}\right|_{\mathrm{L}^{2}(\mathrm{I})}^{2}|\mathrm{u}(\mathrm{a})|_{\mathrm{H}_{0}^{2}}
\end{aligned}
$$

and hence

$$
\left|<\mathrm{u}(\mathrm{a})-\mathrm{z}^{0}, \xi(\mathrm{a} ; \stackrel{0}{\mathrm{~h})}\rangle_{\mathrm{H}_{0}^{2}}\right|<8 \mathrm{~K}_{1}^{3}\left|\mathrm{u}(\mathrm{a})-\mathrm{z}^{0}\right|_{\mathrm{H}_{0}^{2}}|\mathrm{f}|\left|\mathrm{h}_{\mathrm{x}}\right|_{\mathrm{L}^{2}(\mathrm{I})}^{2}
$$

$$
\left|u\left(a_{z^{0}}\right)-z^{0}\right|_{H_{0}^{2}}<\frac{K_{1}^{-2}\left(\frac{k_{1}^{2}}{2}-(\beta-\alpha) k_{2}^{2}\right)}{8 K_{1}^{3}|f|}
$$

Since $a \rightarrow u(a)$ from $Q_{a d}^{2}$ endowed with the $H^{1}$-topology to $H^{2}$ is continuous (observe that $u_{x x}=\frac{1}{a}\left(c u-f-a_{x} u_{x}\right)$ ), there exists $r \in\left(0, r_{1}\right]$ such that (3.17) holds uniformly for all $a \in Q_{a d}^{2}$ with $\left|a-a_{z^{0}}\right| \leq r$. From (3.15) and (3.16) we deduce that there exists a constant $k_{1}>0$ such that

$$
\left.\ln (\mathrm{a} ; \stackrel{0}{\mathrm{~h}})\right|_{\mathrm{H}_{0}^{1}} ^{2}+\left\langle\mathrm{u}(\mathrm{a})-\mathrm{z}^{0}, \xi(\mathrm{a} ; \stackrel{0}{\mathrm{~h}})\right\rangle_{\mathrm{H}_{0}^{1}} \geq \mathrm{k}_{1}\left|\mathrm{~h}_{\mathrm{x}}\right|_{L^{2}(\mathrm{I})}^{2}
$$

for all $a \in Q_{a d}^{2}$ with $\left|a-a_{z^{0}}\right|_{L^{2}(1)} \leq r$ and all $h \in Q$, provided that (3.17) holds. With (H2) holding, the mean value theorem implies that $\alpha=0$ and $\beta=1$ cannot occur simultaneously.

Hence $\left|h_{x}\right|_{L^{2}(\mathrm{I})}^{2} \geq k_{2}|h|_{H^{1}(\mathrm{I})}^{2}$ for some $k_{2}>0$ and all $h \in Q$ and (3.14) follows from (3.18).

The assertion of Theorem 3.2(ii) now follows with the same arguments as in (iv). This ends the proof. 
Remark 3.4 In the definition of $\tilde{Q}_{a d}^{2}$ we chose $m=\int_{\alpha}^{\beta} \hat{a} d x$. This choice which was made for convenience, effects only step (ii) of the proof. If $m \neq \int_{\alpha}^{\beta} \hat{a} d x$ and the additional assumption

$$
|\pi(v-\hat{a})|_{H^{1}(\mathrm{I})}^{2}+\left(m-\int_{\alpha}^{\beta} \hat{a} d x\right)^{2}\left|h_{0}\right|_{H^{1}(I)}^{2}<\gamma^{2}
$$

is made, then again Lemma 2.10 is applicable in step (ii) of the proof and Theorem 3.2 remains valid.

Proof of Theorem 3.3

(i) First some facts are summarized which will be used further below.

There exist constants $\mathrm{K}_{1}, \mathrm{~K}_{2}$ such that the solution $\mathrm{u}(\mathrm{c})$ of (3.2) satisfies

$$
1 / \mathrm{K}_{1}|\mathrm{f}|_{\mathrm{L}^{2}} \leq|\mathrm{u}(\mathrm{c})|_{\mathrm{H}_{0}^{2}} \leq \mathrm{K}_{1}|\mathrm{f}|_{\mathrm{L}^{2}}
$$

and

$$
1 / \mathrm{K}_{2}|\mathrm{f}|_{\mathrm{H}^{-1}} \leq|\mathrm{u}(\mathrm{c})|_{\mathrm{H}_{0}^{1}} \leq \mathrm{K}_{2}|\mathrm{f}|_{\mathrm{H}^{-1}}
$$

for all $c \in Q_{a d}$. For $c \in L^{2}(0,1), c \geq 0$, the mapping $c \rightarrow u(c)$ is twice continuously differentiable from $L^{2}(0,1)$ to $H^{2}(0,1)$ and the first derivative $u_{c}(c)(h)$ in direction $h \in L^{2}(0,1)$, respectively the second derivative $u_{c c}(c)(h, h)$ in direction $(h, h) \in L^{2}(0,1) \times L^{2}(0,1)$ are characterized by

$$
A(c) \eta=-h u(c) \text { and } A(c) \xi=-2 h \eta
$$

where $\eta=\eta(c ; h)=u_{c}(c)(h)$ and $\xi=\xi(c ; h)=u_{c c}(c)(h, h)$ and $A(c): H_{0}^{1} \cap H^{2} \rightarrow L^{2}$ is given by $A(c) \varphi=-\left(a \varphi_{x}\right)_{x}+c \varphi$.

(ii) Next it is shown that $Q_{a d}$ is regular. Let

$$
\mathrm{K}=\left\{\varphi \in \mathrm{L}^{2}(\mathrm{I}): \varphi \geq 0 \text { a.e. }\right\} \times \mathbf{R}^{+}
$$

and let $g: L^{2}(I) \rightarrow L^{2}(I) \times R$ be defined by

$$
g(c)=\left(\left.v|I-c,| c\right|_{L^{2}(I)} ^{2}-\gamma^{2}\right)
$$

Then we find that $Q_{a d}=\left\{\varphi \in L^{2}(I): g(\varphi) \in-K\right\}$.

Since by assumption $|\mathrm{V}|_{\mathrm{L}^{2}}<\gamma$, Lemma 2.10 and Rernark 2.11 implies that every element of $Q_{a d}$ is a regular point.

(iii) To prove the assertion of Theorem 3.3 we employ Theorem 2.5 with $f: Q_{a d} \rightarrow H_{0}^{i}$, $i=1$ or 2 given by 
$f(c)=\left|u(c)-z^{0}\right|_{H_{0}^{i}}^{2}$,

and verify (SSC) with $M(x)=|x|_{H^{i-2}(I)}$. By Proposition 2.1 and (ii) there exists $y^{*}=\left(y_{1}^{*}, y_{2}^{*}\right) \in L^{2} \times R$ satisfying (2.4). Since $g_{c c}(c)(h, h)=\left(0,|h|_{L^{2}}^{2}\right)$ and since $y_{2}^{*} \geq 0$, it suffices
to show the existence of $k_{1}>0$. to show the existence of $k_{i}>0$ and $r_{i}>0$ such that

$$
f_{c c}(c)(h, h) \geq k_{i}|h|_{H^{i-2}(I)}^{2},
$$

for all $c \in Q_{a d}$ with $\left|c-c_{z^{0}}\right|_{H^{i-2(I)}} \leq r_{i}$ and all $h \in L^{2}(I)$ for (SSC) to hold.
Observe that

$$
\mathrm{f}_{\mathrm{cc}}(\mathrm{c})(\mathrm{h}, \mathrm{h})=2|\eta(\mathrm{c} ; \mathrm{h})|_{\mathrm{H}_{0}^{\mathrm{i}}}^{2}+2<\mathrm{u}(\mathrm{c})-\mathrm{z}^{0}, \xi(\mathrm{c} ; \mathrm{h}){ }_{\mathrm{H}_{0}^{\mathrm{i}}}
$$

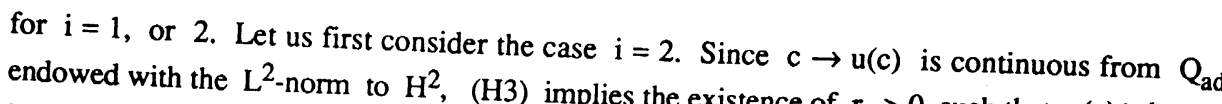
endowed with the $L^{2}$-norm to $H^{2}$, (H3) implies the existence of $r_{3}>0$ such that $u(c) \geq k$ on $[\alpha, \beta]$ for all $c \in Q_{a d}$ with $\left|c-c_{z^{0}}\right|_{L^{2}(1)} \leq r_{3}$. For these $c$ and $h \in L^{2}(I)$ we find by (3.19)
and (3.21)

$$
\begin{aligned}
|\eta(\mathrm{c})(\mathrm{h})|_{\mathrm{H}_{0}^{2}} & =\left|\mathrm{A}^{-1}(\mathrm{c}) \stackrel{0}{\mathrm{~h} u(c)}\right|_{\mathrm{H}_{0}^{2}} \geq\left.\mathrm{K}_{1}^{-1}\right|_{\left.\mathrm{h} \mathrm{u}(\mathrm{c})\right|_{\mathrm{L}^{2}}} \\
& \geq\left.\mathrm{K}_{1}^{-1}||_{\mathrm{hu}}^{0} \mathrm{u}(\mathrm{c})\right|_{\mathrm{L}^{2}(\mathrm{I})} \geq \mathrm{kK}_{1}^{-1} \mid \mathrm{h}_{\mathrm{L}^{2}(\mathrm{I})} .
\end{aligned}
$$

Next observe that for $h \in L^{2}(I)$ and $c \in Q_{a d}$

and therefore

$$
\begin{aligned}
& \left|<\mathrm{u}(\mathrm{c})-\mathrm{z}^{0}, \xi(\mathrm{c} ; \stackrel{0}{\mathrm{~h}})>\right|_{\mathrm{H}_{0}^{2}} \leq 2\left|\mathrm{u}(\mathrm{c})-\mathrm{z}^{0}\right|_{\mathrm{H}_{0}^{2}}\left|\mathrm{~A}^{-1}(\mathrm{c}) \stackrel{0}{\mathrm{~h}} \eta(\mathrm{c} ; \stackrel{0}{\mathrm{~h}})\right|_{\mathrm{H}_{0}^{2}} \\
& \leq 2 \mathrm{~K}_{1}\left|\mathrm{u}(\mathrm{c})-\mathrm{z}^{0}\right|_{\mathrm{H}_{0}^{2}}|\mathrm{~h} \eta(\mathrm{c} ; \mathrm{h})|_{\mathrm{L}^{2}(\mathrm{I})} \\
& \leq 2 \mathrm{~K}_{1}\left|\mathrm{u}(\mathrm{c})-\mathrm{z}^{0}\right|_{\mathrm{H}_{0}^{2}} \cdot|\eta(\mathrm{c} ; \mathrm{h})|_{\mathrm{H}_{0}^{2}}|\mathrm{~h}|_{\mathrm{L}^{2}(\mathrm{I})} \\
& =2 \mathrm{~K}_{1}\left|\mathrm{u}(\mathrm{c})-\mathrm{z}^{0}\right|_{\mathrm{H}_{0}^{2}}\left|\mathrm{~A}^{-1}(\mathrm{c}) \stackrel{0}{\mathrm{~h} u(c)}\right|_{\mathrm{H}_{0}^{2}}|\mathrm{~h}|_{\mathrm{L}^{2}(\mathrm{I})} \\
& \leq 2 \mathrm{~K}_{1}^{2}\left|\mathrm{u}(\mathrm{c})-\mathrm{z}^{0}\right|_{\mathrm{H}_{0}^{2}}|\mathrm{hu}(\mathrm{c})|_{\mathrm{L}^{2}(\mathrm{I})}|\mathrm{h}|_{\mathrm{L}^{2}(\mathrm{I})}
\end{aligned}
$$

(3.24) $\left.\quad\left|<\mathrm{u}(\mathrm{c})-\mathrm{z}^{0}, \xi(\mathrm{c} ; \mathrm{h})\right\rangle_{\mathrm{H}_{0}^{2}}\left|\leq 2 \mathrm{~K}_{1}^{2} \mathrm{~K}_{2}\right| \mathrm{f}\right|_{\mathrm{H}^{-1}}\left|\mathrm{u}(\mathrm{c})-\mathrm{z}^{0}\right|_{\mathrm{H}_{0}^{2}}|\mathrm{~h}|_{\mathrm{L}^{2}(\mathrm{I})}^{2}$.

Let us assume that

$$
\left|\mathrm{u}\left(\mathrm{c}_{\mathrm{z}^{0}}\right)-\mathrm{z}^{0}\right|_{\mathrm{H}_{0}^{2}}<\frac{\mathrm{k}^{2}}{2 \mathrm{~K}_{1}^{4} \mathrm{~K}_{2}|\mathrm{f}|_{\mathrm{H}^{-1}}},
$$

and observe that there exists $r_{2} \in\left(0, r_{3}\right]$ such that (3.25) with $c_{z^{0}}$ replaced by $c$ holds uniformly for all $c \in Q_{a d}$ with $\left|c-c_{z^{0}}\right|_{L^{2}(I)} \leq r_{2}$. 
From (3.23) and (3.24) we deduce the existence of a constant $k_{2}>0$ such that

$$
2|\eta(c ; \stackrel{0}{h})|_{\mathrm{H}_{0}^{2}}^{2}+2<\mathrm{u}(\mathrm{c})-\mathrm{z}^{0}, \xi(\mathrm{c} ; \stackrel{0}{\mathrm{~h}}){ }_{\mathrm{H}_{0}^{2}} \geq \mathrm{k}_{2}|\mathrm{~h}|_{\mathrm{L}^{2}(\mathrm{I})}^{2}
$$

for all $c \in Q_{a d}$ with $\left|c-c_{z^{0}}\right|_{L^{2}(1)} \leq r_{2}$ and all $h \in L^{2}(I)$, provided that (3.25) holds.

This is (3.22) for $\mathrm{i}=2$.

We turn to the case $\mathrm{i}=1$. Since $\mathrm{c} \rightarrow u(c)$ is continuous from $\mathrm{Q}_{\mathrm{ad}}$ endowed with the $\mathrm{H}^{-1}$-norm to $\mathrm{H}^{1}$, there exists $\mathrm{r}_{4}>0$ and $\varepsilon>0$ such that $\mathrm{u}(\mathrm{c}) \geq \mathrm{k}$ on $\mathrm{J}:=[\max (0, \alpha-\varepsilon), \min (1, \beta+\varepsilon)]$ for all $c \in Q_{a d}$ with $\left.\left|\left(c-c_{z^{0}}\right)\right|_{\mathrm{I}}\right|_{\mathrm{H}^{-1}(\mathrm{I})} \leq \mathrm{r}_{4}$. For these $c$ and $\mathrm{h} \in \mathrm{L}^{2}(\mathrm{I})$ we have by (3.20)

$$
\begin{aligned}
& |\eta(c ; \stackrel{0}{h})|_{\mathrm{H}_{0}^{1}}=\left|\mathrm{A}^{-1}(\mathrm{c}) \stackrel{0}{h} \mathrm{u}(\mathrm{c})\right|_{\mathrm{H}_{0}^{1}} \geq\left.\left.\mathrm{K}^{-1}\right|_{\mathrm{h} u(c)} ^{0}\right|_{\mathrm{H}^{-1}}
\end{aligned}
$$

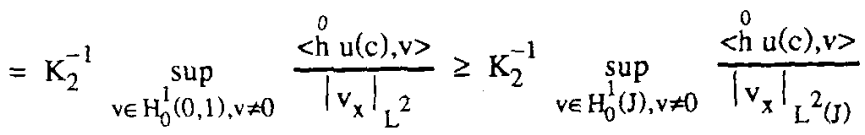

$$
\begin{aligned}
& =\mathrm{K}_{2}^{-1} \sup _{\mathrm{w} \in \mathrm{B}(\mathrm{c}), \mathrm{w \neq 0}} \frac{\stackrel{0}{\mathrm{~h}, \mathrm{w}\rangle}\rangle_{L^{2}(\mathrm{~J})}}{\left|\left(\frac{\mathrm{w}}{\mathrm{u}(\mathrm{c})}\right)_{\mathrm{x}}\right|_{L^{2}(\mathrm{~J})}}
\end{aligned}
$$

where $B(c)=\left\{w u(c): w \in H_{0}^{1}(J)\right\}$. Since $B(c)=H_{0}^{1}(J)$ we find

$$
\mid \eta\left(c ;\left.\stackrel{0}{h)}\right|_{H^{1}} \geq K_{2}^{-1} \sup _{v \in H_{0}^{1}(J), v \neq 0} \frac{\left\langle h^{0}, v\right\rangle_{L^{2}(J)}}{\left|\left(\frac{v}{u(c)}\right)_{x}\right|_{L^{2}(J)}} .\right.
$$

A short calculation gives

$$
\left|\left(\frac{v}{u(c)}\right)_{x}\right|_{L^{2}(J)} \leq \frac{2 K_{2}|f| H^{-1}}{k^{2}}\left|v_{x}\right|_{L^{2}(J)}
$$

and therefore

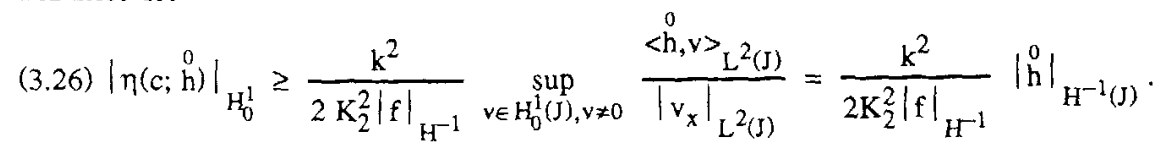

Next observe that for $h \in L^{2}(I)$ and $c \in Q_{a d}$

$\left.(3.27)<\mathrm{u}(\mathrm{c})-\mathrm{z}^{0}, \xi(\mathrm{c} ; \stackrel{0}{\mathrm{~h}})\right\rangle_{\mathrm{H}_{0}^{1}} \leq\left|\mathrm{u}(\mathrm{c})-\mathrm{z}^{0}\right|_{\mathrm{H}_{0}^{1}}\left|\mathrm{~A}^{-1}(\mathrm{c})(\stackrel{0}{\mathrm{~h}} \eta)\right|_{\mathrm{H}_{0}^{1}} \leq \mathrm{K}_{2}\left|\mathrm{u}(\mathrm{c})-\mathrm{z}^{0}\right|_{\mathrm{H}_{0}^{1}}|\stackrel{0}{h} \eta|_{\mathrm{H}^{-1}}$,

where $\eta=\eta(\mathrm{c} ; \stackrel{0}{h})$. In the following estimate the supremum is taken over $0 \neq v \in \mathrm{H}_{0}^{1}$ : 


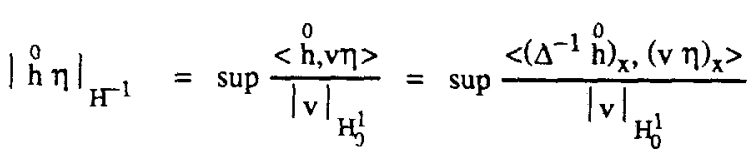

$$
\begin{aligned}
& \leq \sup \frac{\left|\Delta^{-1} \stackrel{0}{h}\right|_{\mathrm{H}_{0}^{1}}\left|(v \eta)_{\mathrm{x}}\right|}{|\mathrm{v}|_{\mathrm{H}_{0}^{1}}} \leq \sup \frac{\left.\left|\Delta^{-1} \stackrel{0}{\mathrm{~h}}\right|_{\mathrm{H}_{0}^{1}}\left|v_{\mathrm{x}} \eta\right|+\left|v \eta_{\mathrm{x}}\right|\right)}{|\mathrm{v}|_{\mathrm{H}_{0}^{1}}} \\
& \leq\left. 2\left|\mathrm{~h}_{\mathrm{H}^{-1}}\right| \eta\right|_{\mathrm{H}_{0}^{1}}=2\left|\mathrm{~h}_{\mathrm{H}^{1}}\right| \mathrm{A}^{-1}(\mathrm{c}) \mathrm{hu} \mathrm{u}_{\mathrm{H}_{0}^{1}} \\
& \leq 2 \mathrm{~K}_{2}|\stackrel{0}{\mathrm{~h}}|_{\mathrm{H}^{-1}}|\stackrel{0}{\mathrm{hu}}|_{\mathrm{H}^{-1}} \leq 4 \mathrm{~K}_{2}|\stackrel{0}{\mathrm{~h}}|_{\mathrm{H}^{-1}}^{2}|\mathrm{u}|_{\mathrm{H}_{0}^{1}} \leq 4 \mathrm{~K}_{2}^{2}|\mathrm{f}|_{\mathrm{H}^{-1}} \mid \stackrel{h}{h}_{\mathrm{H}^{-1}}^{2} \text {, }
\end{aligned}
$$

with $\Delta$ the Laplacian as defined in the proof of Theorem 3.2. Next we prove that there exists $x>0$ such that

$$
\left|h_{h}^{0}\right|_{H^{-1}} \leq \kappa|\stackrel{0}{h}|_{H^{-1}(J)} \text { for all } h \in L^{2}(I)
$$

It suffices to show that

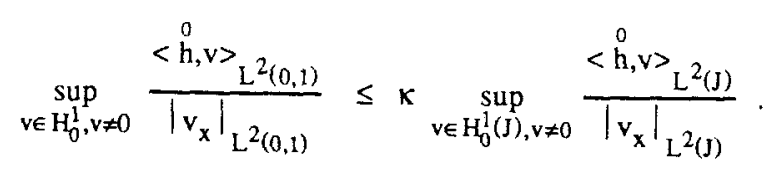

Take any $v \in H_{0}^{l}(0,1)$ and define $\tilde{v} \in H_{0}^{l}(J)$ by

$$
\tilde{v}(x)=\left\{\begin{array}{l}
0 \text { on } I^{c} \\
v \text { on } \mathrm{I} \\
v\left(\frac{\alpha}{\varepsilon} \mathrm{t}+\frac{\alpha}{\varepsilon}(\varepsilon-\alpha)\right) \text { on }(\alpha-\varepsilon, \alpha] \\
v\left(\frac{1-\beta}{\varepsilon} \mathrm{t}+\frac{\beta}{\varepsilon}(\beta-1+\varepsilon)\right) \text { on }[\beta, \beta+\varepsilon) .
\end{array}\right.
$$

Then a straightforward computation shows

$$
\int_{\alpha-\varepsilon}^{\beta+\varepsilon}\left|\tilde{v}_{x}\right|^{2} d x \leq \max \left(\frac{\alpha}{\varepsilon}, 1, \frac{1-\beta}{\varepsilon}\right) \int_{0}^{1}\left|v_{x}\right|^{2} d t
$$

and hence the assertion follows observing that $\langle\mathrm{h}, \mathrm{v}\rangle_{L^{2}(0,1)}=\langle\mathrm{h}, \mathrm{v}\rangle_{L}{ }^{2}(\mathrm{~J})$.

From (3.27) and (3.28) we obtain for all $h \in L^{2}(I)$ and $c \in Q_{a d}$

$$
\left\langle u(c)-z^{0}, \xi(c ; h)\right\rangle_{H_{0}^{1}} \leq 4 K_{2}^{3}|f|_{H^{-1}} \kappa\left|u(c)-z^{0}\right|_{H_{0}^{1}}|h|_{H^{-1}(J)}^{2} .
$$


Let us assume that

$$
\left|\mathrm{u}\left(\mathrm{c}_{\mathrm{z}^{0}}\right)-\mathrm{z}^{0}\right|_{\mathrm{H}_{0}^{1}}^{2}<\frac{\mathrm{k}^{4}}{8 \kappa \mathrm{K}_{2}^{5}|\mathrm{f}|_{\mathrm{H}^{-1}}^{2}} .
$$

Note that there exists $r_{1} \in\left(0, r_{4}\right)$ such that (3.30) with $c_{z^{0}}$ replaced by $c$ holds for all $c \in Q_{a d}$ with $\left|c-c_{z^{0}}\right|_{H^{-1}(\mathrm{I})} \leq r_{1}$. From (3.26) and (3.29) we deduce the existence of a constant $k_{1}>0$ such that

$$
2|\eta(c ; h)|_{\mathrm{H}_{0}^{1}}^{2}+2\left\langle u(c)-z^{0}, \xi\left(\mathrm{c} ; \mathrm{h}^{\circ}\right)\right\rangle_{\mathrm{H}_{0}^{1}} \geq\left.\mathrm{k}_{1}||_{\mathrm{h}}^{0}\right|_{\mathrm{H}^{-1}(\mathrm{~J})} ^{2} \geq \mathrm{k}_{1}|\mathrm{~h}|_{\mathrm{H}^{-1}(\mathrm{I})}^{2}
$$

for all $\mathrm{c} \in \mathrm{Q}_{\mathrm{ad}}$ with $\left|c-\mathrm{c}_{\mathrm{z}^{0}}\right|_{\mathrm{H}^{-1}(1)} \leq \mathrm{r}_{1}$ and all $\mathrm{h} \in \mathrm{L}^{2}(\mathrm{I})$ provided that (3.29) holds.

This is (3.22) for $\mathrm{i}=1$ and the proof is complete.

\section{Remark 3.5}

In the proofs of Theorem 3.2 and 3.3 we ignored the possibility that $y_{2}^{*}$ could be positive; compare (3.12). The effect on the stability of estimating $c$ from data $z$ when $y_{2}^{*}>0$ has been considered in [CK 1; Theorem 5.3]. Of course, $y_{2}^{*}>0$ is special case and it requires that the norm bound in the admissible parameter set is active.

\section{Stability for Regularized Problems}

In this section we consider the problem of estimating the diffusion coefficient a in the Dirichlet problem

$$
\begin{gathered}
-\operatorname{div}(\operatorname{agrad} u)+c u=f \text { on } \Omega \\
u=0 \text { on } \partial \Omega
\end{gathered}
$$

from a measurement $\mathrm{z}$ by means of a regularized least squares fit-to-data criterion. Here $\Omega$ is a bounded domain in $\mathbf{R}^{\mathrm{n}}, \mathrm{n}=2$ or 3 , with a smooth $\left(C^{2}\right.$ )boundary $\partial \Omega$ or $\Omega$ is a parallelepiped, and $f$ and $c$ are fixed elements of $L^{2}$ with $c \geq 0$ and $f \neq 0$. All function spaces are taken over the domain $\Omega$ in this section. The set of admissible coefficients is chosen to be

$$
Q_{a d}=\left\{a \in H^{2}: a \geq v,|a|_{H^{2}} \leq \gamma\right\},
$$

where $v \in \mathrm{H}^{2}, \min v>0$ and $|v|_{\mathrm{H}^{2}}<\gamma$. As a regularization term the square of a seminorm on $\mathrm{H}^{2}$ given by

$$
N(a)=\sum_{i_{1}, j_{2}=1}^{n}\left|a_{x_{i_{1}} x_{i_{2}}}\right|^{2}+\sum_{i_{1}=1}^{n}\left|a_{x_{i_{1}}}\right|^{2}
$$


is used. For a measurement $z \in \mathrm{L}^{2}$ the regularized least squares formulation for estimation of a from $z$ is given by

$(P)_{z}^{\beta} \quad \min |u(a)-z|^{2}+\beta N(a)$ over $a \in Q_{a d}$,

where $\beta$ is a small positive parameter. Since $Q_{a d}$ is a weakly sequentially compact subset of $H^{2}$ and since both $a \rightarrow|u(a)-z|^{2}$ and $a \rightarrow N(a)$ are weakly lower semicontinuous from $H^{2}$ to $R$ it is simple to argue existence of a solution $\mathrm{a}_{\mathrm{z}}^{\beta}$ of $(P)_{z}^{\beta}$ as well as of a solution $\mathrm{a}_{\mathrm{z}}$ of the unregularized problem $(P)_{z}$ (this is $(P)_{2}^{\beta}$ with $\beta=0$ ). It will follow from the proof of Theorem 4.2 that for a certain range of regularization parameters the norm bound is not required for $(P)_{z}^{\beta}$ to have a solution. The norm bound, however, is necessary to guarantee existence of a solution of $(\mathrm{P})_{\mathrm{z}}$. Without it the solutions of $(\mathrm{P})_{\mathrm{z}}^{\beta}$ may diverge as $\beta \rightarrow 0^{+}$.

We shall study the continuous dependence of global and local solutions of $(P)_{z}^{\beta}$ on the observation $z$. Some additional terminology and notation will be used. Let $\mathrm{z}^{0} \in \mathrm{L}^{2}$ denote the unperturbed reference observation. The attainable set $V$ is defined by $V=\left\{u(a): a \in Q_{a d}\right\}$ and for $\beta>0$ we put

$$
\mathrm{Q}^{\beta}=\left\{\mathrm{a}_{\mathrm{z}^{0}}^{\beta} \in \mathrm{Q}_{\mathrm{ad}}: \mathrm{a}_{\mathrm{z}^{0}}^{\beta} \text { is a global solution of }(\mathrm{P})_{\mathrm{z}^{0}}^{\beta}\right\}
$$

and

$$
Q^{0}=\left\{a_{z^{0}} \in Q_{a d}: a_{z^{0}} \text { is a global solution of }(P)_{z^{0}} \text { and } N\left(a_{z^{0}}\right) \text { is minimal }\right\} \text {. }
$$

Clearly the sets $Q^{\beta}$ are not empty. Convergence properties for the solutions of $(P)^{\beta}{ }^{\beta}$ to those of $(P)_{z^{0}}$ are summarized first. In particular it will be shown that $\mathrm{Q}^{0}$ is not empty. All the proofs of the results of this section are given at the end of this section.

\section{Proposition 4.1}

(i) Let $\left\{\beta_{n}\right\}$ be an arbitrary sequence in $R$ with $\beta_{n} \rightarrow 0^{+}$. Then every sequence $\left\{a_{z^{0}}^{\beta_{n}}\right\}$ with $a_{z^{0}}^{\beta_{n}} \in Q^{\beta_{n}}$ has a strong accumulation point in $H^{2}$. Every weak accumulation point is a strong accumulation point and it is contained in $Q^{0}$.

(ii) For every $\varepsilon>0$ there exists $\beta^{+}(\varepsilon)>0$ such that for every $\beta \in\left(0, \beta^{+}(\varepsilon)\right)$ and every $a_{z^{0}}^{\beta} \in Q^{\beta}$ there exists a solution $a_{z^{0}}$ of $(P)_{z^{0}}$ in $Q^{0}$ with

$$
\left|\mathrm{a}_{\mathrm{z}^{0}} \beta^{0}-\mathrm{a}_{\mathrm{z}^{0}}\right|_{\mathrm{H}^{2}}<\varepsilon \text {. }
$$

(iii) The function $\rho(\beta)=N\left(\mathrm{a}_{\mathrm{z}^{0}}\right)-\sup \left\{N\left(\mathrm{a}_{\mathrm{z}^{0}}{ }^{\beta}\right): \mathrm{a}_{\mathrm{z}^{\beta}}{ }_{0} \in Q^{\beta}\right\}$, where $\mathrm{a}_{\mathrm{z}^{0}} \in Q^{0}$, tends to 0 monotonically as $\beta$ tends to $0^{+}$and for all $\beta>0$ and all $a_{z}^{\beta} \in Q^{\beta}$

$$
\left|u\left(a_{z}^{\beta}\right)-z^{0}\right|^{2} \leq \beta \rho(\beta)+\operatorname{dist}\left(z^{0}, V\right)^{2} .
$$

Observe that $N\left(a_{z^{0}}\right)$ is independent of the choice of $a_{z^{0}} \in Q^{0}$ so that $\rho(\beta)$ is welldefined on $(0, \beta)$. In the statement of the following results $K \geq 1$ denotes a constant which depends on $\min v, \gamma, c$, 
$f, \Omega, n$ and the embedding constants of $\mathrm{H}^{2}$ into $\mathrm{L}^{\infty}$ and $\mathrm{H}^{1}$ into $\mathrm{L}^{4}$, and this dependence could, in principle, be calculated explicitly.

Theorem 4.2 (i) Let $\bar{\beta} \leq 1$ be chosen such that

$$
\rho(\bar{\beta})<\frac{1}{K},
$$

let $r>0$ be arbitrary and define

$$
\beta=\frac{\operatorname{dist}\left(z^{0}, V\right)^{2}+r^{2} K}{K^{-1}-\rho(\bar{\beta})}
$$

If $\beta(=\beta(r)) \leq \bar{\beta}$ then there exists a neighborhood $V\left(z^{0}\right)=\left\{z:\left|z-z^{0}\right| \leq \delta_{1}(r)\right\}$ of $z^{0}$ in $L^{2}$ such that for every $\beta \in[\beta, \bar{\beta}], z \in V\left(z^{0}\right)$ and every global solution $a_{z}^{\beta}$ of $(P)_{z}^{\beta}$ there exists $a_{z^{0}}^{\beta} \in Q^{\beta}$ with

$$
\left|a_{z}^{\beta}-a_{z^{0}}^{\beta}\right|_{\infty} \leq\left(\frac{2 L}{\alpha}\right)^{1 / 2}\left|z-z^{0}\right|_{L^{2}}^{1 / 2}
$$

with $\alpha=\left(K^{-1}-\rho(\bar{\beta})\right)(\beta-\beta)$ and $L=2\left(K+\left|z^{0}\right|\right)+1$. If $z^{0} \in V$ then $\beta=r^{2} K\left(K^{-1}-\rho(\bar{\beta})\right)^{-1}$ can be taken arbitrarily small.

(ii) Conversely, for every $\beta \in[\beta, \bar{\beta}]$, every $a_{z^{0}}^{\beta} \in Q^{\beta}$ and every $z \in V\left(z^{0}\right)$ there exists at least one local solution $a_{z}^{\beta}$ of $\left(\mathrm{P}_{\mathrm{z}}^{\beta}\right)$ in $\mathrm{V}_{\infty}\left(\mathrm{a}_{\mathrm{z}}^{\beta}, \mathrm{r}\right)=\left\{\mathrm{a}:\left|\mathrm{a}_{\mathrm{z}} \mathrm{a}_{\mathrm{z}}{ }^{\beta}\right|_{\infty} \leq \mathrm{r}\right\}$ and every such local solution in $\mathrm{V}_{\infty}\left(\mathrm{a}_{\mathrm{z}}{ }_{0}, \mathrm{r}\right)$ satisfies

$$
\left|a_{z}^{\beta}-a_{z^{0}}^{\beta}\right|_{\infty} \leq\left(\frac{2 L}{\alpha}\right)^{1 / 2}\left|z-z^{0}\right|^{1 / 2} \text {. }
$$

As a corollary to the proof of Theorem 4.2 we obtain:

Theorem 4.3 In the notation of Theorem 4.2, if $\beta(r)<\bar{\beta}$, then there exists for every $\beta \in[\beta, \bar{\beta}]$, for every $a_{z^{0}}^{\beta} \in Q^{\beta}$ and every $z \in V\left(z^{9}\right)$ at least one local solution $a_{z}^{\beta}$ of $(P)_{z}^{\beta}$ in $\mathrm{V}_{\mathrm{H}^{2}}\left(\mathrm{a}_{\mathrm{z}^{0}}^{\beta}, \mathrm{r}\right)=\left\{\mathrm{a}:\left|\mathrm{a}-\mathrm{a}_{\mathrm{z}^{0}}^{\beta}\right|_{\mathrm{H}^{2}} \leq \mathrm{r}\right\}$ and every such local solution in $\mathrm{V}_{\mathrm{H}^{2}}\left(\mathrm{a}_{\mathrm{z}^{0}}^{\beta}, \mathrm{r}\right)$ satisfies

$$
\left|a_{z}^{\beta}-a_{z^{0}}^{\beta}\right|_{H^{2}} \leq\left(\frac{2 L}{\alpha}\right)^{1 / 2}\left|z-z^{0}\right| 1 / 2
$$

Corollary 4.4 In the notation of Theorem 4.2, if $\underline{\beta}:=\frac{\operatorname{dist}\left(z^{0}, V\right)^{2}}{K^{-1}-\rho(\bar{\beta})}<\bar{\beta}$, then $(P)_{z^{0}}^{\beta}$ can only have finitely many solutions $a_{z^{0}}^{\beta}$ for every $\beta \in(\underline{\beta}, \bar{\beta}]$.

Remark 4.5 A weaker form of Theorem 4.3 was proved in [CK2]. There the full $\mathrm{H}^{2}$-norm as opposed to the seminorm $N$ was used for regularization, the neighborhoods of $a_{z^{0}}^{\beta}$ within which 
Hölder continuous dependence is asserted were allowed to depend on the specific global solutions $\mathrm{a}_{\mathrm{z}}^{\beta}{ }^{0}$ and only existence of some Hölder constant was given, whereas in (4.2) the dependence of this constant on parameters of $(P)_{z^{0}}^{\beta}$ is clarified.

Observe that the radius $\delta_{1}(r)$ is determined in Corollary 2.7. If the radius $r$ of the ball on which (SSC) holds decreases, so does $\delta_{1}(r)$. In fact we may assume that $\delta_{1}(\cdot)$ is monotonically increasing, left continuous with $\delta_{1}(r)>0$ for $r>0$ and $\delta_{1}(0)=0$.

We note the following lemma.

Lemma 4.6 Suppose that $\mathrm{g}:[0, \infty) \rightarrow[0, \infty)$ is monotonically increasing and left continuous with $g(0)=0$ and $g(x)>0$ for $x>0$. Then there exists a strictly increasing and continuous function $k:[0, \infty) \rightarrow[0, \infty)$ with $0 \leq k(x) \leq g(x)$ for all $x \geq 0$ and $k(x)>0$ for $x>0$.

Hence there exists a continuous and strictly increasing function $\delta_{2}(\cdot)$ with the properties that $\delta_{2}(0)=0$ and $\delta_{2}(r) \in\left(0, \delta_{1}(r)\right\}$ for $r>0$. Since Corollary 2.7 and Theorem 4.2 remain valid if $\delta_{1}(\cdot)$ is decreased, we may assume that $\delta_{1}(\cdot)$ itself is continuous and strictly increasing with $\delta_{1}(0)=0$. In particular there exists a continuous and strictly increasing function $r_{1}(\cdot)$, the inverse of $\delta_{1}(\cdot)$ with $r_{1}(0)=0$ of $\delta_{1}(\cdot)$ with $r_{1}(0)=0$. Observe that $r_{1}(\cdot)$ is defined on the range of $\delta_{1}$ which is of the form $[0, \delta)$ with $\hat{\delta} \in(0, \infty]$. In the next corollary it is tacitly assumed that $\delta \in(0, \hat{\delta})$.

Corollary 4.7 Let $0<\bar{\beta} \leq 1$ be chosen such that $K \rho(\bar{\beta})<1$, let $\varepsilon, \delta$ be positive constants and suppose that $z \in L^{2}$ satisfies $\left|z-z^{0}\right| \leq \delta$. Define

$$
\beta^{-}(\varepsilon, \delta)=\frac{2 L}{K^{-1}-\rho(\bar{\beta})} \cdot \frac{\delta}{\varepsilon^{2}} .
$$

Then for all $\beta \in\left[\beta^{-}(\varepsilon, \delta)+\beta\left(r_{1}(\delta)\right)\right.$, min $\left.\left\{\beta^{+}(\varepsilon), \bar{\beta}\right\}\right]$ and for all global solutions $a_{z}^{\beta}$ of $(P)_{z}^{\beta}$ there exists a solution $a_{z^{0}} \in Q^{0}$ of $(P)_{z^{0}}$ with

$$
\left|a_{z}^{\beta}-a_{z^{0}}\right|_{\infty} \leq\left(1+K_{2}\right) \varepsilon
$$

where $\mathrm{K}_{2}$ is the embedding constant of $\mathrm{H}^{2}$ into $\mathrm{L}^{\infty}$.

Remark 4.8 The range of admissible regularization parameters for the applicability of Corollary 4.7 is given explicitly by

$$
\left[\left(2 \mathrm{~L} \delta / \varepsilon^{2}+\left(\operatorname{dist}\left(z^{0}, V\right)\right)^{2}+\mathrm{r}_{1}(\delta)^{2} \mathrm{~K}\right) /\left(\mathrm{K}^{-1}-\rho(\bar{\beta})\right), \min \left\{\beta^{+}(\varepsilon), \bar{\beta}\right\} .\right.
$$

In particular, if $\mathrm{z}^{0} \in V$, this interval is nonvoid provided that the measurement error $\delta$ is small compared to the desired accuracy $\varepsilon$ for the estimated parameter. 


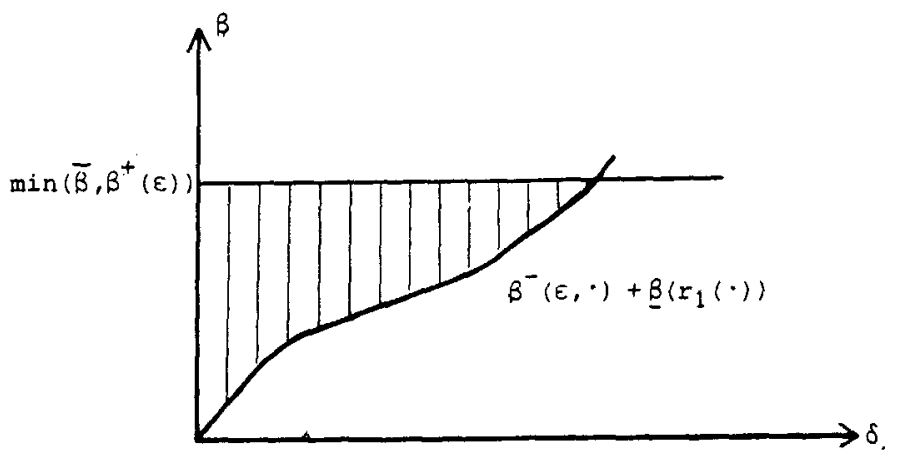

Figure 4.1 ( $\varepsilon$ fixed)

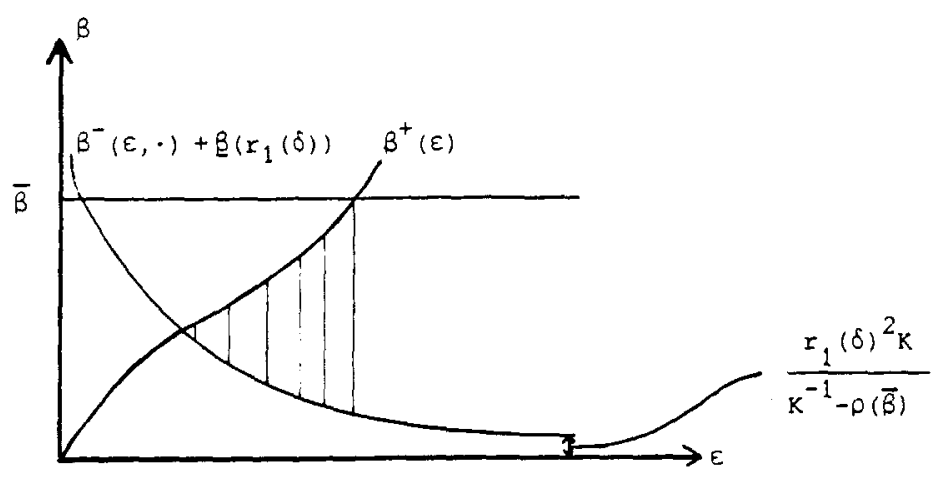

Figure 4.2 (\& fixed)

To demonstrate further Corollary 4.7 we illustrate in Figures 4.1 and 4.2 the range of $\beta$ values for which (4.3) holds in case that $z^{0} \in V$. In Figure 4.1 the admissible $\beta$ values are shown as a function of $\delta$ with $\varepsilon>0$ fixed and Figure 4.2 gives the admissible $\beta$ radius as a function of $\varepsilon$ while $\delta>0$ is fixed.

Remark 4.9 Under the assumption that $u(a)=z$ has a unique solution a result comparable to Corollary 4.7 was obtained with a different method in [KS].

Proof of Proposition 4.1 Part (i) follows with minor modifications from Lemma 3.3 in [CK2]. In [CK2] $N$ was assumed to be radially unbounded. This assumption was only used for existence of solutions of the regularized problems. In the present case this follows from the fact that $Q_{a d}$ is norm bounded. Lemma 3.3 in $[C K 2]$ asserts that $\left\{a_{z^{0}}^{\beta_{n}}\right\}$ has a weak accumulation point as $\mathrm{n} \rightarrow \infty$, that every weak accumulation point is a (global) solution of $(P)_{z^{0}}$ and that $\lim _{n} N\left(a_{z^{0}}^{\beta_{n}}\right)$ exists with $\lim _{n} N\left(a_{z^{0}}^{\beta_{n}}\right)=\min \left\{N\left(a_{z^{0}}\right): z^{0}\right.$ is a global solution of $\left.(P) z^{0}\right\}$. 
Let $\left\{a_{z^{0}}^{\beta_{k}}\right\}$ be a weakly convergent subsequence of $\left\{a_{z^{0}}^{\beta_{k}}\right\}$ with limit $a^{0}$. Then $\left\{a_{z^{0}}^{\beta_{k}}\right\}$ converges strongly to $a^{0}$ in $L^{2}$ and, since $\lim _{k} N\left(a_{z^{0}}^{\beta_{k}}\right)=N\left(a_{z^{0}}\right)=N\left(a^{0}\right), \lim _{k}\left|a_{z^{0}}^{\beta_{k}}\right|_{H^{2}}=\left|a^{0}\right|_{H^{2}}$.

Together with weak convergence of $\left\{a_{z^{0}}^{\beta_{k}}\right\}$ this implies strong convergence of $\left\{a_{z^{0}}^{\beta_{k}}\right\}$ to $a^{0}$ in $H^{2}$ and ends the proof of (i).

To verify (ii) suppose that for some $\varepsilon>0$ and for every $n \in N$ there exists $\beta_{n} \in(0,1 / n)$ and $a_{z^{0}}^{\beta_{n}} \in Q^{\beta_{n}}$ such that for all solutions $a_{z^{0}}$ of $(P)_{z^{0}}$ in $Q^{0}$

$$
\left|\mathrm{a}_{\mathrm{z}^{0}}^{\beta_{\mathrm{n}}}-\mathrm{a}_{\mathrm{z}^{0}}\right|_{\mathrm{H}^{2}} \geq \varepsilon \text {. }
$$

By (i) one can choose $\left\{a_{z^{0}}^{\beta_{n}}\right\}$ such that this sequence converges strongly to a solution of $(P) z^{0}$ in $Q^{0}$. This is a contradiction to (4.4).

Finally (iii) follows from

$$
\left|u\left(a_{z^{0}}^{\beta}\right)-z^{0}\right|^{2}+\beta N\left(a_{z^{0}}^{\beta}\right) \leq\left|u\left(z_{z^{0}}\right)-z^{0}\right|^{2}+\beta N\left(a_{z^{0}}\right),
$$

which holds for all $\beta>0, a_{z^{0}} \in Q^{\beta}$ and $a_{z^{0}} \in Q^{0}$.

Proof of Theorem 4.2 The first part of this theorem will be verified by demonstrating the applicability of Corollary 2.7 in conjunction with Remark 2.8 . To establish the connection with Corollary 2.7 let $D=\left\{a \in H^{2}: a>\min v\right\}$, let $W=L^{2}$ and define $f^{\beta}: D \times W \rightarrow R$ by

$$
f^{\beta}(a, z)=|u(a)-z|^{2}+\beta N(a)
$$

The X-topology of $Q_{a d} \subset D$ is defined by the $L^{\infty}$-topology and $Q=H^{2}$. Since $Q_{a d}$ is a weakly sequentially compact subset of $\mathrm{H}^{2}$ and since $\mathrm{H}^{2}$ embeds continuously into $\mathrm{L}^{\infty}$, (i) and (ii) of Corollary 2.7 are satisfied and (iii) can be verified by a short calculation. It remains to establish assumption (iv) of Corollary 2.7 and the continuity assumption of $f$ specified in Remark 2.8. Some technical preliminaries are summarized first.

For $a \in Q_{a d}$ let $A(a)$ denote the realization in $L^{2}$ of the differential operator in (4.1); i.e. $\operatorname{dom} \mathrm{A}(\mathrm{a})=\mathrm{H}^{2} \cap \mathrm{H}_{0}^{1}$ and $\mathrm{A}(\mathrm{a}) \varphi=-\operatorname{div}(\mathrm{a} \operatorname{grad} \varphi)+c \varphi$. Observe that $\mathrm{u}(\mathrm{a})=\mathrm{A}^{-1}$ (a)f gives the solution of (4.1), that $A(a)$ is selfadjoint and that $A(a)$ is a homeomorphism from $\mathrm{H}^{2} \cap \mathrm{H}_{0}^{1}$ onto $\mathrm{L}^{2}$ for every $\mathrm{a} \in \mathrm{Q}_{\mathrm{ad}}$. There exists $\mathrm{K}_{1}>0$ depending on $\min v, \gamma, \mathrm{c}$ and $\Omega$ such that

$$
\frac{1}{K_{1}}|g|_{L^{2}} \leq\left|A^{-1}(a) g\right|_{H^{2}} \leq K_{1}|g|_{L^{2}}
$$

for every $g \in L^{2}$ and $a \in Q_{a d}$. From (4.5) we deduce the inequalities 
(4.6)

$$
\frac{1}{K_{1}}|g|_{H^{-2}} \leq \mid A^{-1}\left(\text { a) }\left.g\right|_{L^{2}} \leq K_{1}|g|_{H^{-2}}\right.
$$

for every $g \in L^{2}$ and $a \in Q_{a d}$. Indeed, using (4.5) we have

$$
\begin{aligned}
\left|A^{-1}(a) g\right| & =\sup _{0 \neq v \in L^{2}} \frac{\left(A^{-1}(a) g, v\right)}{|v| L_{L^{2}}}=\sup _{0 \neq v \in L^{2}} \frac{\left(g, A^{-1}(a) v\right)}{|v|_{L^{2}}} \\
& =\sup _{0 \neq v \in H^{2} \cap H_{0}^{1}} \frac{(g, v)}{|A(a) v|_{L^{2}}} \geq \frac{1}{K_{1}} \sup _{0 \neq v \in H^{2} \cap H_{0}^{1}} \frac{(g, v)}{|v| H_{H^{2}}}=|g|_{H^{-2}} .
\end{aligned}
$$

The second inequality in (4.6) is proved analogously. Moreover $K_{1}$ can also be chosen sufficiently large so that

$$
\frac{1}{\mathrm{~K}_{1}}|\mathrm{~g}|_{\mathrm{L}^{2}} \leq\left|\Delta^{-1} \mathrm{~g}\right|_{\mathrm{H}^{2}} \leq \mathrm{K}_{1}|\mathrm{~g}|_{\mathrm{L}^{2}}
$$

holds for all $\mathrm{g} \in \mathrm{L}^{2}$. From (4.7) we obtain $\left|\Delta^{-1} \mathrm{~g}\right| \leq \mathrm{K}_{1}|\mathrm{~g}|_{\mathrm{H}^{-2}}$ for all $\mathrm{g} \in \mathrm{L}^{2}$ and hence with (4.6) we find

$$
\frac{1}{\mathrm{~K}_{1}}|\mathrm{f}|_{\mathrm{H}^{-2}} \leq|\mathrm{u}(\mathrm{a})| \leq \mathrm{K}_{1}|\Delta \mathrm{u}(\mathrm{a})|_{\mathrm{H}^{-2}}
$$

and therefore

$$
\frac{1}{\mathrm{~K}_{\mathrm{l}}^{2}}|\mathrm{f}|_{\mathrm{H}^{-2}} \leq|\Delta \mathrm{u}(\mathrm{a})|_{\mathrm{H}^{-2}} \text { for every } \mathrm{a} \in \mathrm{Qda}_{\mathrm{ad}}
$$

Since $n=2$ or 3 there exist constants $K_{2}$ and $K_{3}$ such that

$$
|\mathrm{h}|_{\infty} \leq \mathrm{K}_{2}|\mathrm{~h}|_{\mathrm{H}^{2}}
$$

and

$$
|h|_{L^{4}} \leq K_{3}|h|_{H^{2}}
$$

for all $h \in H^{2}$. We recall that $|h|_{\infty}$ is used for $|h|_{L}$.

To verify the Lipschitz continuity of $f$ specified in Remark 2.8 , let $a \in Q_{a d}$ and $z \in V\left(z^{0}, 1\right)=\left\{z \in L^{2}:\left|z^{0}-z\right| \leq 1\right\}$. We find

$$
\begin{aligned}
\left|f^{\beta}\left(a, z^{0}\right)-f^{\beta}(a, z)\right| & =|\operatorname{lu}(a)-z|+\left|u(a)-z^{0}\right||||u(a)-z|-\left|u(a)-z^{0}\right| \mid \\
& \leq\left(2\left|u(a)-z^{0}\right|+\left|z-z^{0}\right|\right)\left|z-z^{0}\right| \\
& \leq\left[2\left(K_{1}|f|+\left|z^{0}\right|\right)+1\right]\left|z-z^{0}\right|
\end{aligned}
$$


and thus $\mathrm{f}^{\beta}$ is Lipschitz continuous with respect to $z$ uniformly in a (and independently of $\beta$ ) and the Lipschitz constant can be chosen as $L=2\left(K+\left|z^{0}\right|\right)+1$, where $K$ has the properties specified before Theorem 4.2 .

Turning to assumption (iv) of Corollary 2.7 let us first observe that by Lemma 2.10 and Remark 2.11 every element of $Q_{a d}$ is regular. Therefore there exists for every $a_{z^{0}}^{\beta} \in Q^{\beta}$ a Lagrange multiplier $\lambda^{*}=\left(\lambda_{1}^{*}, \lambda_{2}^{*}\right) \in H^{2} \times \mathbf{R}$ satisfying (2.4). In particular $\lambda_{2}^{*} \geq 0$. Here we suppressed the dependence of $\lambda^{*}$ on the specific solution $a_{z^{0}}^{\beta}$. The Lagrange functional associated with $(P)_{z^{0}}^{\beta}$ is given by

$$
F(a)=\left|u(a)-z^{0}\right|^{2}+\lambda^{*} g(a)+\beta N(a)
$$

where $g: H^{2} \rightarrow H^{2} \times R$ is defined by $g(a)=\left(v-a,|a|_{H^{2}}^{2}-\gamma^{2}\right)$.

The second derivative of $F$ w.r.t. a is given by

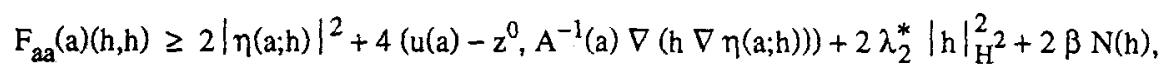

where

$$
\eta(a ; h)=A^{-1}(a) \nabla(h \nabla u(a))
$$

(c.f. [CK2, (4.12), (4.13) and Example 5.2]). It will be convenient to drop the dependence on $a$ in the notation of $\eta(a ; h)$. Using the facts that $\lambda_{2}^{*} \geq 0$, that $-a b \geq-a^{2}-\frac{b^{2}}{4}$ for any $a$ and $b$ in $\mathbf{R}$ and the selfadjointness of $A(a)$ we find

$$
F_{a a}(a)(h, h) \geq 2|\eta(h)|^{2}+4\left(\nabla\left(h \nabla\left(A^{-1}(a)\left(u(a)-z^{0}\right)\right)\right), \eta(h)\right)+2 \beta N(h)
$$

$$
\geq|\eta(h)|^{2}-4\left|\nabla\left(h \nabla\left(A^{-1}(a)\left(u(a)-z^{0}\right)\right)\right)\right|^{2}+2 \beta N(h) .
$$

Henceforth $K \geq 1$ is used as a generic constant depending only on $K_{1}, K_{2}, K_{3}, n$ and $f$. By (4.5), (4.7), (4.9) and (4.10) we obtain

$$
\begin{aligned}
& \left|\nabla\left(h \nabla\left(A^{-1}(a)\left(u(a)-z^{0}\right)\right)\right)\right| \leq\left|h \Delta\left(A^{-1}(a)\left(u(a)-z^{0}\right)\right)\right|+\left|\nabla h \cdot \nabla\left(A^{-1}(a)\left(u(a)-z^{0}\right)\right)\right| \\
& \quad \leq K_{1}|h|_{\infty}\left|A^{-1}(a)\left(u(a)-z^{0}\right)\right|_{H^{2}}+\sum_{i=1}^{n}\left|h_{x_{i}}\right|_{L^{4}}\left|\left(A^{-1}(a)\left(u(a)-z^{0}\right)\right)_{x_{i}}\right|_{L^{4}} \\
& \quad \leq K_{1}^{2} K_{2}|h|_{H^{2}}\left|u(a)-z^{0}\right|+n K_{1} K_{3}^{2}|h|_{H^{2}}\left|u(a)-z^{0}\right| \leq K|h|_{H^{2}}\left|u(a)-z^{0}\right|
\end{aligned}
$$

and thus by (4.12)

$$
F_{a a}(a)(h, h) \geq 2 \ln \left(h_{1}\right)+\left.\eta\left(h_{2}\right)\right|^{2}-4 K|h|_{H^{2}}^{2}\left|u(a)-z^{0}\right|^{2}+2 \beta N(h),
$$

where $h \in H^{2}$ has been decomposed as $h=h_{1}+h_{2}$ with 


$$
h_{1}=\int_{\Omega} h(x) d x \text { and } h_{2} \in\left\{\varphi \in H^{2}: \int_{\Omega} \varphi(x) d x=0\right\} .
$$

For every $h \in \mathrm{H}^{2}$ we find by (4.5)

$$
\begin{aligned}
|\eta(h)| & =\left|A^{-1}(a)(\nabla h \nabla u(a))\right|=\sup _{0 \neq v \in L^{2}} \frac{\left(\nabla(h \nabla u(a)), A^{-1}(a) v\right)}{|v|} \\
& =\sup _{0 \neq v \in H^{2} \cap H_{0}^{1}} \frac{(\nabla(h \nabla u(a)), v)}{|A(a) v|} \leq K_{1} \sup _{0 \neq v \in H^{2} \cap H_{0}^{1}} \frac{(h \nabla u(a), \nabla v)}{|v|_{\mathrm{H}^{2}}} \\
& \leq K_{1} \sup _{0 \neq v \in \mathrm{H}^{2} \cap H_{0}^{1}} \frac{\left(\Sigma\left|h u_{x_{1}}(a)\right|_{L^{2}}^{2}\right)^{1 / 2}\left(\Sigma\left|v_{x_{i}}\right|_{L^{2}}^{2}\right)^{2}}{|v|_{H^{2}}} \\
& \leq K_{1}|h|_{\infty}|\mathrm{u}(a)|_{H^{2}} \leq K_{1}^{2} K_{2}|h|_{H^{2}}|f|
\end{aligned}
$$

and hence

$$
|\eta(h)|^{2} \leq \mathrm{K}|\mathrm{h}|_{\mathrm{H}^{2}}^{2} \text { for every } \mathrm{h} \in \mathrm{H}^{2}
$$

For a constant function with value $h_{1}$ one obtains with (4.6) and (4.8)

$$
\left|\eta\left(h_{1}\right)\right|=\left|A^{-1}(a) h_{1} \Delta u(a)\right| \geq \frac{\left|h_{1}\right|}{K_{1}}|\Delta u(a)|_{H^{-2}} \geq \frac{\left|h_{1}\right|}{K_{1}^{3}}|f|_{H^{-2}}
$$

and hence

$$
\left|\eta\left(h_{1}\right)\right|^{2} \geq \frac{1}{K}\left|h_{1}\right|_{L^{2}}
$$

From [IK2] we recall that

$$
N\left(h_{2}\right) \geq K\left|h_{2}\right|_{H^{2}}^{2}
$$

for any $h_{2} \in\left\{\varphi \in \mathrm{H}^{2}: \int \varphi d x=0\right\}$.

Using (4.14) with $h=h_{2},(4.15)$ and (4.16) in (4.13) we find for every $\kappa>1$

$$
\begin{aligned}
\mathrm{F}_{\mathrm{aa}}(\mathrm{a})(\mathrm{h}, \mathrm{h}) & \geq \frac{\kappa-1}{\kappa}\left|\eta\left(\mathrm{h}_{1}\right)\right|^{2}+(1-\kappa)\left|\eta\left(\mathrm{h}_{2}\right)\right|^{2}-4 \mathrm{~K}\left(\left|\mathrm{~h}_{1}\right|_{\mathrm{L}^{2}}^{2}+\left|\mathrm{h}_{2}\right|_{\mathrm{H}^{2}}^{2}\right)\left|\mathrm{u}(\mathrm{a})-\mathrm{z}^{0}\right|^{2}+2 \beta \mathrm{N}(\mathrm{h}) \\
& \geq\left[\frac{\kappa-1}{\kappa} \frac{1}{\mathrm{~K}}-4 \mathrm{~K}\left|\mathrm{u}(\mathrm{a})-\mathrm{z}^{0}\right|^{2}\right]\left|\mathrm{h}_{1}\right|_{\mathrm{L}^{2}}^{2}+\left[2 \beta+(1-\kappa) \mathrm{K}-4 \mathrm{~K}\left|\mathrm{u}(\mathrm{a})-\mathrm{z}^{0}\right|^{2}\right]\left|\mathrm{h}_{2}\right|_{\mathrm{H}^{2}}^{2}
\end{aligned}
$$


For $\beta \in(0,1]$ choose $x=1+\frac{\beta}{K}$. It follows that $\kappa-1=\frac{\beta}{K}, \frac{1}{K}=\frac{K}{K+\beta} \geq \frac{K}{K+1} \geq \frac{1}{2}$ and

$$
\begin{aligned}
F_{a a}(a)(h, h) & \geq\left[\frac{\beta}{2 K^{2}}-4 K\left|u(a)-z^{0}\right|^{2}\right]\left|h_{1}\right|_{L^{2}}^{2}+\left[\beta-4 K\left|u(a)-z^{0}\right|^{2}\right]\left|h_{2}\right|_{H^{2}}^{2} \\
& \geq \beta\left[\frac{1}{2 K^{2}}-4 K \frac{\left|u(a)-z^{0}\right|^{2}}{\beta}\right]\left(\left|h_{1}\right|^{2}+\left|h_{2}\right|_{H^{2}}^{2}\right) \\
& \geq 8 K^{2} \beta\left[\frac{1}{\left(8 K^{2}\right)^{2}}-\frac{\left|u(a)-z^{0}\right|^{2}}{2 \beta}\right]|h|_{H^{2}}^{2}
\end{aligned}
$$

Thus we find

$$
F_{a a}(a)(h, h) \geq \beta\left[\frac{1}{K}-\frac{\left|u(a)-z^{0}\right|^{2}}{2 \beta}\right] \min \left(|h|_{H^{2}}^{2},|h|_{\infty}^{2}\right)
$$

for some generic constant $K \geq 1$ uniformly in $\beta \in(0,1]$ and $a \in Q_{a d}$.

Using the mean value theorem one can show that $q \rightarrow u(q)$ is globally Lipschitz continuous from $Q_{a d}$ endowed with the $L^{\infty}$-topology to $L^{2}$ (compare the estimate before (4.14)), so that $K$ can be chosen such that

$$
|u(a)-u(a)|_{L^{2}} \leq \sqrt{K} \min \left(|a-\tilde{a}|_{H^{2}},|a-\tilde{a}|_{\infty}\right)
$$

for all $a$ and $\bar{a}$ in $Q_{a d}$. As in the statement of the theorem let $r>0$ and recall that $\beta$ and $\bar{\beta}$ are chosen such that

$$
\rho(\bar{\beta})=N\left(a_{z^{0}}\right)-\sup \left\{N\left(a_{2^{0}} \bar{\beta}_{z^{0}} \in \mathbf{a}^{\bar{\beta}}\right\}<\frac{1}{K},\right.
$$

where $a_{z^{0}} \in Q^{0}$ and

$$
B=\eta^{-1}\left(\operatorname{dist}\left(z^{0}, V\right)^{2}+r^{2} K\right)
$$

where we put $\eta=K^{-1}-\rho(\bar{\beta})$.

From Proposition 4.1 it is known that

$$
\left|u\left(a_{z^{0}}^{\beta}\right)-z^{0}\right|^{2} \leq \beta \rho(\beta)+\operatorname{dist}\left(z^{0}, V\right)^{2} .
$$

With (4.17) - (4.19) we obtain for all $a \in B=\left\{a:\left|a-a_{z^{0}}^{\beta}\right|_{\infty} \leq r, a_{z^{0}}^{\beta} \in Q^{\beta}, \beta \in[\beta, \bar{\beta}]\right\}$ and all $h \in L^{\infty}$

$$
F_{a a}(a)(h, h) \geq \beta\left[\frac{1}{K}-\frac{\left|u(a)-u\left(a_{z^{0}}^{\beta}\right)\right|^{2}}{\beta}-\frac{\left|u\left(a_{z^{0}}^{\beta}\right)-z^{0}\right|^{2}}{\beta}\right]|h|_{\infty}^{2}
$$




$$
\begin{aligned}
& \geq \beta\left[\frac{1}{\mathrm{~K}}-\frac{\mathrm{r}^{2} \mathrm{~K}}{\beta}-\rho(\bar{\beta})-\frac{\operatorname{dist}\left(z^{0}, V\right)^{2}}{\beta}\right]|\mathrm{h}|_{\infty}^{2} \\
& =\beta\left[\eta-\frac{\beta \eta}{\beta}\right]|\mathrm{h}|_{\infty}^{2}=\eta(\beta-\beta)|\mathrm{h}|_{\infty}^{2} .
\end{aligned}
$$

This is (iv) of Corollary 2.7 with $\alpha=\left(K^{-1}-p(\bar{\beta})\right)(\beta-\beta)$ uniformly for all $a_{z^{0}}^{\beta} \in Q^{\beta}$ and $\beta \in[\beta, \bar{\beta}]$. Reconsidering the proof of Corollary (2.7) one observes that $V\left(z^{0}\right)$ can be chosen independently of $\beta \in[\beta, \bar{\beta}]$ and the first part of Theorem 4.2 is proved. Theorem 4.2 (ii) follows from Theorem 2.5, Remark 2.6 and (4.20).

Proof of Theorem 4.3 From (4.17) - (4.19) it follows with a calculation analogous to (4.20) that

$$
F_{a \mathrm{a}}(\mathrm{a})(\mathrm{h}, \mathrm{h}) \geq\left(\frac{1}{\mathrm{~K}}-\rho(\bar{\beta})\right)(\beta-\beta)|\mathrm{h}|_{\mathrm{H}^{2}}^{2}
$$

for all $h \in H^{2}$ and all $a \in\left\{a:\left|a-a_{z^{0}}^{\beta}\right|_{H^{2}} \leq r, a_{z^{0}}^{\beta} \in Q^{\beta}, \beta \in[\beta, \bar{\beta}]\right\}$. An application of Theorem 2.5 together with Remark 2.6 gives the desired result.

Pro of of Corollary 4.4 Assume that the cardinality of $Q^{\beta}$ is infinite for some $\beta \in(\beta, \bar{\beta})$. Then $r$ can be chosen such that $\beta \in[\beta(r), \bar{\beta}]$ so $r^{\mathrm{l}}$ ut Theorem 4.2 (ii) is applicable. Let $\left\{a_{z^{0}}^{\beta, n}\right\}$ be a nontrivial sequence in $Q^{\beta}$. Since $\left\{\mathrm{a}_{\mathrm{z}^{0}}^{\beta, n} \mathrm{H}^{2^{2}}\right\}$ is bounded there exists a subsequence of $\left\{\mathrm{a}_{\mathrm{z}^{0}}^{\beta, \mathrm{n}}\right\}$ converging strongly in $L^{\infty}$. This contradicts Theorem 4.2 (ii) from which it follows that the global solutions $a_{z^{0}}{ }_{0}$ are unique in the balls $v_{\infty}\left(a_{z_{0}}^{\beta}, r\right)$.

Proof of Lemma 4.6 It suffices to prove the assertion for $g$ mapping $[0,1]$ into $[0, g(1)]$. Since $g$ is monotone, $\lim _{x \rightarrow 0} g(x)$ exists. If this limit is greater than zero, the assertion follows. If $\lim _{x \rightarrow 0} g(x)=0$, define

$$
x_{n}:=\sup \{x: g(x) \leq 1 / n\} \text { for } n=N_{0}, N_{0}+1, \ldots
$$

where $N_{0}$ is determined by $g(1)>\frac{1}{N_{0}}$. Due to left continuity and monotonicity, the supremum is actually attained. Furthermore $0<\mathrm{g}\left(\mathrm{x}_{\mathrm{n}}\right) \leq 1 / \mathrm{n}$ and $\left\{\mathrm{x}_{\mathrm{n}}\right\}$ is decreasing by definition. There is a strictly decreasing subsequence $\left\{x_{n_{k}}\right\}$ with $\frac{1}{n_{k+1}}<g\left(x_{n_{k}}\right) \leq \frac{1}{n_{k}}$. Then $x_{n_{k}} \rightarrow 0$, since otherwise $x_{n_{k}}$ tends monotonically to some $\alpha>0$ and $0<g(\alpha) \leq g\left(x_{n_{k}}\right) \leq \frac{1}{n_{k}}$ with $n_{k} \rightarrow \infty$. Now define a step function $h(\cdot)$ by

$$
h(x)=g\left(x_{n_{k}}\right) \text { on }\left(x_{n_{k}}, x_{n_{k-1}}\right] \text {. }
$$


By construction $\lim _{x \rightarrow 0} h(x)=0$ and $0<h(x) \leq g(x)$ for all $x>0$. Finally the desired function $k(x)$ is obtained by linear interpolation of $\left(x_{n_{k}}, g\left(x_{n_{k+1}}\right)\right)$ and $\left(x_{n_{k-1}}, g\left(x_{n_{k}}\right)\right)$ on $\left(x_{n_{k}}, x_{n_{k-1}}\right)$.

Proof of Corollary 4.7 We shall apply Proposition 4.1 and Theorem 4.2 (i). For $\beta$ in the interval of admissible regularization parameters, one has $\beta \geq B\left(r_{1}(\delta)\right)$. Hence, applying Theorem 4.2(i) with $r=r_{1}(\delta)$, we obtain that for every global solution $a_{z}^{\beta}$ of $(P)_{z}^{\beta}$ with $\left|z-z^{0}\right| \leq \delta=\delta_{1}(r(\delta))$ there exists a global solution $a_{z^{0}}^{\beta} \in Q^{\beta}$ such that

$$
\left|a_{z}^{\beta}-a_{z^{0}}^{\beta}\right|_{\infty} \leq\left(\frac{2 L}{\left[K^{-1}-p(\bar{\beta})\right]\left[\beta-\beta\left(r_{1}(\delta)\right)\right]}\right)^{1 / 2}\left|z-z^{0}\right|^{1 / 2} \leq \varepsilon
$$

Moreover, Proposition 4.1 implies that for every $a_{z^{0}}^{\beta} \in Q^{\beta}$ there exists a solution $a_{z^{0}}$ of $(P)_{z^{0}}$ in $Q^{0}$ with

$$
\left|a_{z^{0}}^{\beta}-a_{z^{0}}\right|_{\infty} \leq K_{2}\left|a_{z^{0}}^{\beta}-a_{z^{0}}\right|_{H^{2}} \leq K_{2} \varepsilon
$$

Taken together, we find for every global solution $a_{z}^{\beta}$ of $(P)_{z}^{\beta}$ a solution $a_{z^{0}}$ of $(P) z^{0}$ with

$$
\left|\mathrm{a}_{z}^{\beta}-\mathrm{a}_{z^{0}}\right|_{\infty} \leq \varepsilon+\mathrm{K}_{2} \varepsilon \text {. }
$$

This ends the proof of Corollary 4.7.

\section{Stability for Regularized Problems with Small Modelling Errors.}

In Theorem 4.2, stability of the solutions $\mathrm{a}_{z}^{\beta_{0}}$ of the regularized problem $(P)_{z^{0}}^{\beta_{0}}$ with respect to perturbations of $z$ was shown under the a-priori assumption that $\beta<\bar{\beta}$. In this section we first prove a stability property, where "modelling errors" in the system equation are taken into consideration: if these modelling errors are sufficiently small and if the observation is attainable by the model-error free system, then there always exists an interval of regularization parameters for which the regularized problems are stable with respect to perturbations in the observation. Furthermore we analyse the relationship between the perturbed regularized and the unperturbed unregularized problems.

Consider the family of parameter estimation problems

(P) $)_{z ; c, f}^{\beta} \quad$ minimize $|u(a ; c, f)-z|^{2}+\beta N(a)$ over $a \in Q_{a d}$, 
where $u(a ; c, f)$ is a solution of $(4.1)$ and $(c, f)$ are taken from a weakly sequentially compact subset $M$ of $\left\{(\mathrm{c}, f) \in \mathrm{L}^{2} \times \mathrm{L}^{2}: \mathrm{c}(\mathrm{x}) \geq 0\right.$, a.e. $\}$. Recall that this is equivalent to the assumption that $M$ is weakly closed and bounded. It can be checked that the constant $\mathrm{K}$ of section 4 can be chosen uniformly w.r.t. $(c, f) \in M$.

The results of this section depend upon the following attainability assumption, i.e. for the unperturbed observation $z^{0}$ there exist model parameters $\left(a^{0}, c^{0}, f^{0}\right) \in Q_{a d} \times M$ such that

$$
u\left(a^{0} ; c^{0}, f^{0}\right)=z^{0}
$$

The parameter $a^{0}$ is to be estimated, while an estimate $(c, f)$ for $\left(c^{0}, f^{0}\right)$ is assumed to be available and fixed. Replacing $\left(c^{0}, f^{0}\right)$ by $(c, f)$ takes into account the modelling error. We study its influence on the estimation of $a^{0}$ from $(P)_{z ; c, f}^{\beta}$. For $(c, f) \in M$ we call $\quad(P)_{z^{0} ; c, f}^{0}$ the unperturbed, unregularized and $(P)_{z ; c, f}^{\beta}$ the perturbed regularized problem.

Henceforth all neighborhoods of $\left(\mathrm{c}^{0}, \mathrm{f}^{0}\right)$ will be understood to be a subset of $M$ and $\mathrm{K}$ will be uniform w.r.t. $(c, f) \in M$. The solutions of $(\mathrm{P})_{z ; c, f}^{\beta}$ are denoted by $a_{z}^{\beta}(c, f)$.

As in (4.11) one can show that $z \rightarrow l u(a ; c, f)-\left.z\right|^{2}+\beta N(a)$ is globally Lipschitz continuous at $z^{0}$ in the neighborhood $V\left(z^{0}, 1\right) \subset L^{2}$ of the unperturbed observation $z^{0}$, uniformly with respect to $(\mathrm{a} ; \mathrm{c}, \mathrm{f}) \in \mathrm{Q}_{\mathrm{ad}} \times M$. This Lipschitz constant is denoted by $\mathrm{L}$.

Theorem 5.1 Let $z^{0} \in \mathrm{L}^{2}$ and suppose that there are model parameters $\left(a^{0} ; c^{0}, f^{0}\right) \in Q_{a d} \times M$ with

$$
u\left(a^{0} ; c^{0}, f^{0}\right)=z^{0}
$$

Let $\bar{\beta}$ satisfy $\mathrm{K}^{-1}>\rho(\bar{\beta})$ and choose $\beta^{*} \in(0, \bar{\beta})$. Then there are a weak $\mathrm{L}^{2} \times \mathrm{L}^{2}$-neighborhood $\mathrm{V}\left(\mathrm{c}^{0}, \mathrm{f}^{0}\right) \subset M$, a strong $\mathrm{L}^{2}$-neighborhood $\mathrm{V}\left(\mathrm{z}^{0}\right)$ and a neighborhood $\mathrm{V}\left(\beta^{*}\right)$ of $\left(\mathrm{c}^{0}, \mathrm{f}^{0}\right), \mathrm{z}^{0}$ and $\beta^{*}$ respectively such that for every global solution $a_{z}^{\beta}(c, f)$ of $(P)_{z ; c, f}^{\beta}$ with $(z ; c, f ; \beta) \in V\left(z^{0}\right) \times V\left(c^{0}, f^{0}\right) \times V\left(\beta^{*}\right)$, there exists a global solution $a_{z^{0}}^{\beta}(c, f)$ of $(P)_{z^{0} ; c, f}^{\beta}$ with

$$
\left|a_{z}^{\beta}(c, f)-a_{z^{0}}^{\beta}(c, f)\right|_{\infty} \leq 4\left(\frac{L}{\beta^{*}\left(K^{-1}-\rho(\bar{\beta})\right)}\right)^{1 / 2}\left|z-z^{0}\right|^{1 / 2}
$$

Conversely, there exists $r>0$ such that for every global solution $a_{z^{0}}^{\beta}(c, f)$ of $(P)_{z^{0} ; c, f}^{\beta}$ with 
$(c, f, \beta) \in V\left(c^{0}, f^{0}\right) \times V\left(\beta^{*}\right)$ and every $z \in V\left(z^{0}\right)$ there exists a local solution $a_{z}^{\beta}(c, f)$ of (P) $)_{z ; c, f}^{\beta}$ in $V_{\infty}\left(a_{z^{0}}^{\beta}(c, f), I\right)=\left\{a \in Q_{a d}:\left|a-a_{z^{0}}^{\beta}(c, f)\right|_{\infty} \leq I\right\}$ and every such local solution in $V_{\infty}\left(a_{z}(c, f), r\right)$ satisfies $(5.2)$.

Next we analyse the relationship between $(P)_{z^{0} ; c, f}^{\beta}$ and $(P)_{z^{0} ; c, f^{\circ}}^{0}$

Lemma 5.2 Suppose that the model parameter $\left(\mathrm{a}^{0}, \mathrm{c}^{0}, \mathrm{f}^{0}\right)$ satisfying $(5.1)$ is unique and choose $\beta^{+}(\varepsilon)$ according to Proposition 4.1(ii). Then for every $\beta^{*} \in\left(0, \beta^{+}(\varepsilon)\right)$ there exist a neighborhood $\tilde{V}\left(\beta^{*}\right)$ of $\beta^{*}$ and a weak $L^{2} \times L^{2}$ neighborhood $\tilde{V}\left(c^{0}, f^{0}\right)$ of $\left(c^{0}, f^{0}\right)$ such that all global solutions $a_{z^{0}}^{\beta}(c, f)$ of $(P){ }_{z^{0} ; c, f}^{\beta}$ and $a_{z^{0}}^{0}(c, f)$ of $(P){ }_{z^{0} ; c, f}^{0}$ with $(\beta, c, f) \in \tilde{V}\left(\beta^{*}\right) \times \tilde{V}\left(c^{0}, f^{0}\right)$ satisfy

$$
\left|a_{z^{0}}^{\beta}(c, f)-a_{z^{0}}^{0}(c, f)\right|_{\infty} \leq \varepsilon K_{2}
$$

where $K_{2}$ is an embedding constant of $\mathrm{H}^{2}$ into $\mathrm{L}^{\infty}$

To describe an approximation property of $(\mathrm{P})_{z ; c, f}^{\beta}$ to $(\mathrm{P})_{z_{;}^{0}, \mathrm{o}, f}^{0}$ we combine Theorem 5.1 and Lemma 5.2. Choose $\delta_{1}>0$ such that $V\left(z^{0} ; \delta_{1}\right)=\left\{z \in L^{2}:\left|z-z^{0}\right| \leq \delta_{1}\right\} \subset V\left(z^{0}\right)$, with $\mathrm{V}\left(z^{0}\right)$ as in Theorem 5.1. Recall the notation $\beta^{-}(\varepsilon, \delta)=\frac{2 \mathrm{~L}}{\mathrm{~K}^{-1}-\rho(\bar{\beta})} \frac{\delta}{\varepsilon^{2}}$.

Theorem 5.3 Suppose that the model parameter $\left(\mathrm{a}^{0}, \mathrm{c}^{0}, \mathrm{f}^{0}\right) \in \mathrm{Q}_{\mathrm{ad}} \times M$ satisfying (5.1) is unique and choose $\varepsilon>0,0<\delta<\delta_{1}$. Then for every $\beta^{*}$ with $8 \beta^{-}(\varepsilon, \delta)<\beta^{*}<\min \left(\bar{\beta}, \beta^{+}(\varepsilon)\right)$ there are a weak $L^{2} \times L^{2}$-neighborhood $\hat{V}\left(c^{0}, f^{0}\right)$ of $\left(c^{0}, f^{0}\right)$ and a strong $L^{2}$-neighborhood $\hat{V}\left(\beta^{*}\right)$ of $\beta^{*}$, such that for all global solutions $a_{z}^{\beta}(c, f)$ of $(P)_{z, c, f}^{\beta}$ with $(\beta, c, f) \in \hat{V}\left(\beta^{*}\right) \times \hat{V}\left(c^{0}, f^{0}\right)$ and $\left|z-z^{0}\right| \leq \delta$ there exists a global solution $a_{z}^{0}(c, f)$ of $(P)_{z}^{0} ; c, f$ with

$$
\left|a_{z}^{\beta}(c, f)-a_{z}^{0} 0(c, f)\right|_{\infty} \leq\left(1+K_{2}\right) \varepsilon,
$$

where $\mathrm{K}_{2}$ is the embedding constant of $\mathrm{H}^{2}$ into $\mathrm{L}^{\infty}$.

Remark 5.4 For $\delta$ sufficiently small one can always guarantee that $8 \beta^{-}(\varepsilon, \delta)=\frac{16 \mathrm{~L} \delta}{\varepsilon^{2}\left(\mathrm{~K}^{-1}-\rho(\bar{\beta})\right)}<\min \left(\bar{\beta}, \beta^{*}(\varepsilon)\right)$.

Remark 5.5 Consider the one-dimensional boundary value problem

$$
\begin{aligned}
& -\left(a u_{x}\right)_{x}=f, \\
& u(0)=u(1)=0,
\end{aligned}
$$


for $a \in Q_{a d}=\{a \in R: a \in[1,2]\}$. For $f \in L^{2}(0,1)$, put $V(f)=\left\{u(a): a \in Q_{a d}\right\}$. We give an example such that

$$
\left\{\begin{array}{l}
z^{0} \in V\left(f^{0}\right) \\
z^{0} \notin V\left(f_{n}\right), f_{n} \rightarrow f^{0} \text { in } C(0,1) \\
\lim _{n \rightarrow \infty} \operatorname{dist}\left(z^{0}, V\left(f_{n}\right)\right)_{L^{2}}=0 .
\end{array}\right.
$$

Thus for the model-error free system atrainability holds, but arbitrarily close there are systems (i.e. $\left.-\left(a u_{x}\right)_{x}=f_{n}\right)$ for which $z^{0} \&\left(f_{n}\right)$. Properties (5.3) hold for the choice

$$
z^{0}=\pi^{-2} \sin \pi x, f^{0}=\sin \pi x, \text { and } f_{n}=\frac{n}{n+1} \sin \pi x, n=1,2, \ldots
$$

In this case $V\left(f_{n}\right)=\left\{\frac{n}{n+1} \frac{1}{a \pi^{2}} \sin \pi x: a \in[1,2]\right\}$. Clearly $z^{0} \in V\left(f^{0}\right), f_{n} \rightarrow f^{0}$ in $C$ and $z^{0} \notin V\left(f_{n}\right)$ for $n=1,2, \ldots$ hold. Since $z_{n} \rightarrow z^{0}$ in $L^{2}(0,1)$, for $z_{n}=\frac{n}{n+1} \frac{1}{\pi^{2}} \sin \pi x \in V\left(f_{n}\right)$, it follows that $\lim _{n \rightarrow \infty} \operatorname{dist}\left(z^{0}, V\left(f_{n}\right)\right)_{L^{2}}=0$.

Remark 5.6 In this section stability w.r.t. perturbation of the error free observation $z^{0}$ was guaranteed provided that the model error (represented by the distance from $(c, f)$ to $\left.\left(c^{0}, f^{0}\right)\right)$ is sufficiently small. An attainability assumption for the observation - and model-error free system was made throughout.

The techniques of the paper can also be applied to the related problem of stability of the solutions of $(\mathrm{P})_{z ; c, f}^{\beta}$ with respect to perturbations of the observation-model coefficient vector $w=(z ; c, f)$.

Let us consider the attainable case in which for the observation-error free $z^{0}$ there exist a modelerror free coefficient $\left(c^{0}, f^{0}\right)$ and $a^{0} \in Q_{a d}$ such that $u\left(a^{0}, c^{0}, f^{0}\right)=z^{0}$. Define a metric space $W=L^{2} \times W_{1}$, where $W_{1}$ is a weakly compact subset of $L^{2} \times L^{2}$ containing $\left(c^{0}, f^{0}\right)$. In particular, $W_{1}$ is metrizable. Using the notation of Theorem 4.2, if, for $r>0$, $\beta=\frac{r^{2} K}{K^{-1}-\rho(\bar{\beta})} \leq \bar{\beta}$, then there exists $\alpha>0$ and a neighborhood $\bar{V} \subset W$ of $\left(z^{0}, c^{0}, f^{0}\right)$ in the $\mathrm{L}^{2} \times \mathrm{L}_{\text {weak }}^{2} \times \mathrm{L}_{\text {weak }}^{2}$-topology, such that for every global solution $\mathrm{a}_{\mathrm{z}}^{\beta}(\mathrm{c}, \mathrm{f})$ of $(\mathrm{P})_{\mathrm{z} ; \mathrm{c}, \mathrm{f}}^{\beta}$ with

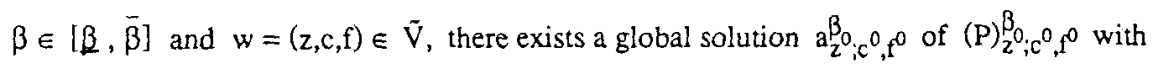

$$
\left|a_{z ; c, f}^{\beta}-a_{z^{0} ; c^{0}, 0}^{\beta}\right|_{\infty} \leq\left(\frac{2}{a}\right)^{1 / 2} \varepsilon\left(\delta\left(w, w^{0}\right)\right)^{1 / 2}
$$

Here $\varepsilon(\cdot)$ is the modulus of continuity of the mapping $(z, c, f) \rightarrow|u(c, f)-z|^{2}$ from $\mathrm{L}^{2} \times \mathrm{L}_{\text {weak }}^{2} \times \mathrm{L}_{\text {weak }}^{2}$ to $\mathrm{R}$.

Proof of Theorem 5.1 We apply Corollary 2.7 together with Remark 2.8. Assumptions (i) (iii) of Corollary 2.7 are shown in the same manner as in the first part of the proof of Theorem 4.2 . 
We turn to (iv) of Corollary 2.7. The Lagrange functional of $(P)_{z_{;}^{0}, f}^{\beta}$ is given by

$$
F(a ; c, f, \beta)=1 u(a ; c, f)-\left.z^{0}\right|^{2}+\lambda^{*} g(a)+\beta N(a),
$$

where $(c, f) \in M, \beta \geq 0, a \in Q_{a d}$ and $g$ is defined as in the proof of Theorem 4.2. Observe that for any solution $a_{z}^{\beta}(c, f)$ the associated Lagrange parameter $\lambda^{*}=\left(\lambda_{1}^{*}, \lambda_{2}^{*}\right)$ satisfies $\lambda_{2}^{*} \geq 0$. Let $\beta^{*} \in(0, p(\bar{\beta}))$. We shall show that there exist $r>0$, a weak $L^{2} \times L^{2}$-neighborhood $V\left(c^{0}, f^{0}\right)$ of $\left(c^{0}, f^{0}\right)$ and a neighborhood $V\left(\beta^{*}\right)$ of $\beta^{*}$ such that

$$
F_{a a}(a ; c, f, \beta)(h, h) \geq \frac{L}{8\left(K^{-1}-\rho(\bar{\beta})\right)}|h|_{\infty}^{2}
$$

for all $h \in H^{2}$ and all $a \in \tilde{B}=\left\{a \in Q_{a d}:\left|a-a_{z^{3}}^{\beta}(c, f)\right|_{H^{2}} \leq r, a_{z^{0}}^{\beta}(c, f)\right.$ a solution of $(P)_{z^{0} ; c, f}^{\beta}$ with $\left.\beta \in V\left(\beta^{*}\right),(c, f) \in V\left(c^{0}, f^{0}\right)\right\}$. This will imply the claim.

As in the proof of Theorem 4.2, see (4.17), one can show that

$$
F_{a a}(a ; c, f, \beta)(h, h) \geq \beta\left[K^{-1}-\frac{\left|u(a ; c, f)-z^{0}\right|^{2}}{\beta}\right]|h|_{\infty}^{2},
$$

for all $h \in \mathrm{H}^{2}, a \in \mathrm{Q}_{\mathrm{ad}}$ and $(c, f) \in M$. Let $\mathrm{a}_{\mathrm{z}^{0}}^{\beta}(\mathrm{c}, \mathrm{f})$ be a solution of $(\mathrm{P})_{\mathrm{z}^{0} ; \mathrm{c}, \mathrm{f}}^{\beta_{0}}$. We find

$$
\begin{aligned}
F_{a a}(a ; c, f, \beta)(h, h) & =\left[\left|u\left(a_{z^{0}}^{\beta}(c, f), c, f\right)-z^{0}\right|^{2}-\left|u(a, c, f)-z^{0}\right|^{2}\right]|h|_{\infty}^{2} \\
& +\left[\frac{\beta}{K}-\left|u\left(a_{z^{0}}^{\beta}(c, f), c, f\right)-z^{0}\right|^{2}|h|_{\infty}^{2}\right. \\
& \geq-2 K\left(\left|u\left(a_{z^{0}}^{\beta}(c, f), c, f\right)-z^{0}\right|-\left|u(a, c, f)-z^{0}\right|\right)|h|_{\infty}^{2} \\
& +\left[\frac{\beta}{K}-\left|u\left(a_{z^{0}}^{\beta}(c, f), c, f\right)-z^{0}\right|^{2}\right]|h|_{\infty}^{2} \\
& \geq-2 K\left|u\left(a_{z^{0}}^{\beta}(c, f), c, f\right)-u(a, c, f)\right||h|_{\infty}^{2} \\
& +\left[\frac{\beta}{K}-\left|u\left(a_{z^{0}}^{\beta}(c, f), c, f\right)-z^{0}\right|^{2}\right]|h|_{\infty}^{2} .
\end{aligned}
$$

Since $\mathrm{a} \rightarrow \mathrm{u}(\mathrm{a} ; \mathrm{c}, \mathrm{f})$ from $\mathrm{Q}_{\mathrm{ad}} \subset \mathrm{H}^{2}$ to $\mathrm{L}^{2}$ is Lipschitz continuous, uniformly with respect to $(\mathrm{c}, \mathrm{f}) \in M$ we have

$$
F_{a a}(a ; c, f, \beta) \geq-2 K^{2}|h|_{\infty}^{2}+\left[\frac{\beta}{K}-\left|u\left(a_{z^{0}}^{\beta}(c, f), c, f\right)-z^{0}\right|^{2}\right]|h|_{\infty}^{2}
$$


for all $a \in \tilde{B}$. By Proposition 4.1 and due to the attainability assumption we find for every solution $a_{z^{0}}^{\beta^{*}}\left(c^{0}, f^{0}\right)$ of $(P)_{z^{0} ; c^{0}, f^{0}}^{\beta^{*}}$

$$
\left[\beta^{*} K^{-1}-\left|u\left(a_{z^{0}}^{\beta^{*}}\left(c^{0}, f^{0}\right), c^{0}, f^{0}\right)-z^{0}\right|^{2}\right]=\beta^{*}\left(K^{-1}-\rho\left(\beta^{*}\right)\right) \geq \beta^{*} \eta
$$

where $\eta=K^{-1}-\rho(\bar{\beta})$. There exists a closed neighborhood $V\left(c^{0}, f^{0}\right) \subset M$ of $\left(c^{0}, f^{0}\right)$ in the weak $\mathrm{L}^{2} \times \mathrm{L}^{2}$ (metrizable) topology on $M$ such that for all solutions $\mathrm{a}_{\mathrm{z}^{0}}^{\beta^{*}}(\mathrm{c}, f)$ of $(\mathrm{P}) \mathrm{z}^{0} ; \mathrm{c}, \mathrm{f}$, with $(\mathrm{c}, \mathrm{f}) \in \mathrm{V}\left(\mathrm{c}^{0}, \mathrm{f}^{0}\right)$

$$
\left[\beta^{*} K^{-1}-\left|\mathrm{u}\left(\mathrm{a}_{z^{0}}^{\beta^{*}}(c, f), c, f\right)-z^{0}\right|^{2}\right] \geq \frac{\beta^{*} \eta}{2}
$$

holds. If this were not the case, then there would exist a sequence $\left\{\left(c_{n}, f_{n}\right)\right.$ in $M$ and solutions $\left\{a_{z^{0}}^{\beta^{*}}\left(c_{n}, f_{n}\right)\right\}$ of $(P){ }_{z^{0}}^{\beta_{0}^{*}} ; c_{n}, f_{n}$ with $c_{n} \rightarrow c^{0}$ and $f_{n} \rightarrow f^{0}$ weakly in $L^{2}$, and

$$
\beta^{*} K^{-1}-\left|u\left(a_{z^{0}}^{\beta^{*}}\left(c_{n}, f_{n}\right), c_{n}, f_{n}\right)-z^{0}\right|^{2}<\frac{\beta^{*} \eta}{2}
$$

Since $\left\{a_{z}^{\beta_{0}^{*}}\left(c_{n}, f_{n}\right)\right\}$ is a bounded sequence in $H^{2}$, it contains a weakly convergent subsequence with limit $\hat{a} \in \mathrm{H}^{2}$. We shall drop the subsequential index. It is simple to show that

$$
u\left(a_{z^{0}}^{\beta^{*}}\left(c_{n}, f_{n}\right), c_{n}, f_{n}\right) \rightarrow u\left(\hat{a}, c^{0}, f^{0}\right) \text { in } L^{2}
$$

and taking the limit in (5.9) this implies

$$
\beta^{*} K^{-1}-\left|u\left(\hat{a}, c^{0}, f^{0}\right)-z^{0}\right|^{2} \leq \frac{\beta^{*} \eta}{2}
$$

It has also simple to show that $\hat{a}$ is a solution of $(P)_{2}^{\beta_{0}^{*}} ; c^{0}, f^{0}$ and hence by (5.7)

$$
\beta^{*} \mathrm{~K}^{-1}-\left|\mathrm{u}\left(\hat{\mathrm{a}}, \mathrm{c}^{0}, \mathrm{f}^{0}\right)-z^{0}\right|^{2} \geq \beta^{*} \eta,
$$

holds. This contradicts (5.10) and hence (5.8) holds. Moreover there exists a neighborhood $\mathrm{V}\left(\beta^{*}\right)$ of $\beta^{*}$ such that

$$
\beta K^{-1}-\left|u\left(a_{z}^{\beta}(c, f), c, f\right)-z^{0}\right|^{2} \geq \frac{\beta^{*} \eta}{4}
$$

for all $\beta \in V\left(\beta^{*}\right)$ and $(c, f) \in V\left(c^{0}, f^{0}\right)$. If this were wrong, there would exist a nontrivial sequence $\left\{\beta_{n}\right\}$ converging to $\beta^{*}$ and solutions $a_{z}{ }^{0}\left(c_{n}, f_{n}\right)$ with $\left(c_{n}, f_{n}\right) \in V\left(q^{0}, z^{0}\right)$ such that

$$
\beta_{n} K^{-1}-\left|u\left(a_{z^{0}}^{\beta_{n}}\left(c_{n}, f_{n}\right), c_{n}, f_{n}\right)-z^{0}\right|^{2}<\frac{\beta^{*} \eta}{4} .
$$


Without loss of generality it can be assumed that

$$
c_{n} \rightarrow \tilde{c}, f_{n} \rightarrow \tilde{f} \text { in weakly } L^{2} \text {; and } a_{z}^{\beta_{n}}\left(c_{n}, f_{n}\right) \rightarrow \tilde{a} \text { weakly in } H^{2},
$$

for some elements $(c, f) \in V\left(c^{0}, f^{0}\right)$ and $\tilde{a} \in Q_{a d}$. Then

$$
u\left(a_{z^{0}}^{\beta_{n}}\left(c_{n}, f_{n}\right), c_{n}, f_{n}\right) \rightarrow u(\bar{a}, \tilde{c}, \tilde{f}) \text { in } L^{2}
$$

and by (5.12)

$$
\beta * \mathrm{~K}^{-1}-\left|\mathrm{u}(\tilde{a}, \tilde{c}, \tilde{\mathrm{f}})-\mathrm{z}^{0}\right|^{2} \leq \frac{\beta^{*} \eta}{4}
$$

But $\bar{a}$ is a solution of $(P)_{z^{0} ; \tilde{c}, \tilde{f}}^{\beta^{*}}$ and thus (5.13) contradicts (5.8). Hence (5.11) holds.

Combining (5.6) and (5.11) we have for $r=\frac{\beta^{*} \eta}{16 \mathrm{~K}^{2}}$

$$
F_{a a}(a ; c, f, \beta)(h, h) \geq\left[-2 K^{2} r+\frac{\beta^{*} \eta}{4}\right]|h|_{\infty}^{2}=\frac{\beta^{*} \eta}{8}|h|_{\infty}^{2},
$$

for all $(c, f, \beta) \in V\left(c^{0}, f^{0}\right) \times V(\beta *), a \in \tilde{B}$ and $h \in H^{2}$.

The second part of Theorem 5.1 follows from Theorem 2.5, Remark 2.6 and (5.14). This ends the proof.

Proof of Lemma 5.2 Choose $\beta^{*} \in\left(0, \beta_{0}(\varepsilon)\right)$ and suppose, contrary to the assertion, that there exist sequences $\left\{\beta_{n}\right\}$ in $R$ and $\left\{\left(c_{n}, f_{n}\right)\right\}$ in $M$ with

$$
\beta_{n} \rightarrow \beta^{*}, \quad\left(c_{n}, f_{n}\right) \rightarrow\left(c^{0}, f^{0}\right) \text { weakly in } L^{2} \times L^{2}
$$

and solutions $a_{z^{0}}^{\beta_{n}}\left(c_{n}, f_{n}\right)$ of $(P)_{z^{0} ; c_{n}, f_{n}}^{\beta_{n}}$ and $a_{z^{0}}^{0}\left(c_{n}, f_{n}\right)$ of $(P)_{z^{0} ; c_{n}, f_{n}}^{0}$ such that

$$
\left|a_{z^{0}}^{\beta_{n}}\left(c_{n}, f_{n}\right)-a_{z^{0}}^{0}\left(c_{n}, f_{n}\right)\right|_{\infty} \geq \varepsilon K_{2}
$$

Since $\left\{a_{z}^{\beta_{n}}\left(c_{n}, f_{n}\right)\right\}$ and $\left\{a_{z^{0}}^{0}\left(c_{n}, f_{n}\right)\right\}$ are bounded sequences in $H^{2}$, there are subsequences (we drop the subsequential index) converging weakly in $H^{2}$ and strongly in $L^{\infty}$ to $\tilde{a}_{z^{0}}^{-\beta^{*}}$ and $\tilde{a}^{0}$ respectively. From (5.15) it follows that

$$
\left|\tilde{\mathrm{a}}_{\mathbf{z}^{0}}^{\beta^{*}}-\tilde{\mathrm{a}}^{0}\right|_{\infty} \geq \varepsilon \mathrm{K}_{2} .
$$

The optimality property of $a_{z^{0}}^{\beta_{n}}\left(c_{n}, f_{n}\right)$ implies

$$
\left|u\left(a_{z^{0}}^{\beta_{n}}\left(c_{n}, f_{n}\right), c_{n}, f_{n}\right)-z^{0}\right|^{2}+\beta_{n} N\left(a_{z^{0}}^{\beta_{n}}\left(c_{n}, f_{n}\right)\right) \leq\left|u\left(a, c_{n}, f_{n}\right)-z^{0}\right|^{2}+\beta_{n} N(a)
$$


for all $a \in Q_{a d^{*}}$. Since weak convergence of $\left(a_{z^{0}}^{\beta_{n}}\left(c_{n}, f_{n}\right), c_{n}, f_{n}\right)$ in $H^{2} \times L^{2} \times L^{2}$ to $\left(a_{z^{0}}^{\beta *}, c^{0}, f^{0}\right)$ implies convergence of $u\left(a_{z^{0}}^{\beta_{n}}\left(c_{n}, f_{n}\right), c_{n}, f_{n}\right)$ to $u\left(\hat{a}_{z^{0}}^{\beta^{*}}, c^{0}, f^{0}\right)$, and due to weak lower semicontinuity of $\mathrm{N}$ from $\mathrm{H}^{2}$ to $\mathbf{R}$ we find from (5.17) $\operatorname{lu}\left(a_{z^{0}}^{\beta^{*}}, c^{0}, f^{0}\right)-\left.z^{0}\right|^{2}+\beta^{*} N\left(a_{z^{0}}^{\beta^{*}}\right) \leq\left|u\left(a, c^{0}, f^{0}\right)-z^{0}\right|^{2}+\beta^{*} N(a)$ for all $a \in Q$ ad. Hence $a_{z^{0}}^{\beta^{*}}$ is a solution of $\left(\mathrm{P}_{z^{\prime} ; c^{0}, \mathrm{p}^{0}}^{\beta^{*}}\right.$. Similarly one can show that $\tilde{a}^{0}$ is a solution of $\left(\mathrm{P}_{z^{0} ; c^{0}, \mathrm{f}^{0}}^{0}\right.$. By the uniqueness assumption $\tilde{a}^{0}=a^{0}$ and Proposition 4.1 implies that

$$
\left|\tilde{a}_{z^{0}}^{\beta^{*}}-\mathrm{a}^{0}\right|_{\infty}<\varepsilon \mathrm{K}_{2} .
$$

This contradicts (5.16) and ends the proof.

Proof of Theorem 5.3 Let $\varepsilon>0$ and choose $\beta^{*}<\min \left(\beta^{+}(\varepsilon), \bar{\beta}\right)$. By Theorem 5.1 and Lemma 5.2 there exist a neighborhood $\hat{V}\left(\beta^{*}\right)$ of $\beta^{*}$ and a weak $L^{2} \times L^{2}$-neighborhood $\hat{V}\left(c^{0}, f^{0}\right)$ of $\left(c^{0}, f^{0}\right)$ such that for all global solutions $a_{z}^{\beta}(c, f)$ of $(P)_{z ; c, f}^{\beta}$ with $(z ; c, f ; \beta) \in V\left(z^{0} ; \delta_{1}\right) \times \hat{V}\left(c^{0}, f^{0}\right) \times \hat{V}\left(\beta^{*}\right)$ there exists a global solution $a_{z^{0}}^{\beta}(c, f)$ of $(P){ }_{z}^{0} ; c, f$ with

$$
\left|a_{z}^{\beta}(c, f)-a_{z^{0}}^{\beta}(c, f)\right|_{\infty} \leq 4\left(\frac{L}{\beta^{*} \eta}\right)^{1 / 2}\left|z-z^{0}\right|^{1 / 2}
$$

and for all global solutions $a_{z^{0}}^{\beta}(c, f)$ of $(P)_{z^{0} ; c, f}^{\beta}$ and $a_{z^{0}}^{0}(c, f)$ of $(P)_{z^{0} ; c, f}^{0}$

$$
\left|\mathrm{a}_{z^{0}}^{\beta}(\mathrm{c}, \mathrm{f})-\mathrm{a}_{\mathrm{z}^{0}}^{0}(\mathrm{c}, \mathrm{f})\right|_{\infty} \leq \varepsilon \mathrm{K}_{2} .
$$

Thus for all global solutions $a_{z}^{\beta}(c, f)$ of $(P)_{z ; c, f}^{\beta}$ with $(z ; c, f ; \beta) \in V\left(z^{0} ; \delta_{1}\right) \times \hat{V}\left(c^{0}, f^{0}\right) \times \hat{V}\left(\beta^{*}\right)$ there exists a global solution $a_{z}^{0}(c, f)$ of $(P)_{2}^{0} ; c, f$ such that

$$
\left|a_{z}^{\beta}(c, f)-a_{z^{0}}^{0}(c, f)\right|_{\infty} \leq 4\left(\frac{L}{\beta * \eta}\right)^{1 / 2}\left|z-z^{0}\right|^{1 / 2}+\varepsilon K_{2} .
$$

If in addition to the above restrictions on $(z ; c, f, \beta)$, we have $\left|z-z^{0}\right| \leq \delta$ and $\beta \in\left(8 \beta^{-}(\varepsilon, \delta)\right.$, $\left.\min \left(\beta, \beta^{+}(\varepsilon)\right)\right)$, then

$$
\left|a_{z}^{\beta}(c, f)-a_{z^{0}}^{0}(c, f)\right|_{\infty} \leq 4\left(\frac{L}{\beta^{*} \eta}\right)^{1 / 2} \delta^{1 / 2}+\varepsilon K_{2} \leq \varepsilon\left(K_{2}+1\right)
$$

This ends the proof.

\section{References}

[Ad] R.A. Adams: Sobolev Spaces, Academic Press, 1975. 
[Al] W. Alt: Lipschitzian perturbations of infinite optimization problems, in: Mathematical Programming with Data Perturbations II, ed. by A.V. Fiacco, Lecture Notes in Pure and Applied Mathematics, 85, Marcel Dekker, New York 1984, 7-21.

[C1] G. Chavent: Local stability of the output least square parameter estimation technique, Matematica Aplicada e Computacional, V.2(1983), 3-22.

[C2] G. Chavent: A geometric approach for the a-priori study of nonlinear inverse problems, Proceedings of the RCP Meeting on Inverse Problems, Montpellier, France, Dec. 1986, $1-13$.

[C3] G. Chavent: On the uniqueness of local minima for general abstract non-linear least square problems, INRIA Report No. 645.

[CK1] F. Colonius and K. Kunisch: Stability for parameter estimation in two point boundary value problems, J. Reine Angewandte Mathematik, 370(1986), 1-29.

[CK2] F. Colonius and K. Kunisch: Output least squares stability in elliptic systems, Applied. Math. and Optimization, 19(1989), 33-63.

[EKN] H.W. Engl, K. Kunisch and A. Neubauer: Tikhonov regularization for the solution of nonlinear ill-posed problems I, submitted to Inverse Problems

[G] P. Grisvard: Elliptic Problems in Nonsmooth Domains, Pitman, Boston 1985.

[IK] K. Ito and K. Kunisch: The augmented Lagrangian method for parameter estimation in elliptic system, to appear in SIAM J. Control and Optimization.

[KS] C. Kravaris and J.H. Seinfeld: Identification of parameters in distributed systems by regularization, SIAM J. Control and Optimization, 23(1985), 217-241.

[LU] C.A. Ladyzhenskaya and N.N. Ural'tseva: Linear and Quasilinear Elliptic Equations, Academic Press 1968.

[M] H. Maurer: First and second order sufficient optimality conditions in mathematical programming and optimal control, Mathematical Programming Study 14(1981), 163-177.

[MZ] H. Maurer and J. Zowe: First and second-order necessary and sufficient optimality conditions for infinite-dimensional programming problems, Mathematical Programming, 16(1979), 98-110.

[N] A. Neubauer: Tikhonov regularization for the solution of nonlinear ill-posed problems II: submitted to Inverse Problems.

[Ri] G.R. Richter: An inverse problem for the steady state diffusion equation, SIAM, J.Appl. Math. 41(1981), 210-221.

[Ru] D.L. Russell: Some remarks on numerical aspects of coefficient identification in elliptic systems, in: Optimal Control of Partial Differential Equations, ed. by K.H. Hoffmann and W. Krabs, Birkhäuser 1984, 210-118.

[W] J. Werner: Optimization Theory and Applications, Vieweg, Braunschweig, 1984. 